\title{
The Main Progress of Perovskite Solar Cells in 2020-2021
}

Cite as

Nano-Micro Lett.

(2021) $13: 152$

Received: 29 April 2021

Accepted: 7 June 2021

Published online: 7 July 2021

(C) The Author(s) 2021

\author{
Tianhao $\mathrm{Wu}^{1}$, Zhenzhen Qin ${ }^{1}$, Yanbo Wang ${ }^{1}$, Yongzhen $\mathrm{Wu}^{2}$, Wei Chen ${ }^{3}$, \\ Shufang Zhang ${ }^{4}$, Molang Cai ${ }^{5}$, Songyuan Dai ${ }^{5}$, Jing Zhang ${ }^{6}$, Jian Liu ${ }^{7}$, Zhongmin Zhou ${ }^{8}$, \\ Xiao Liu ${ }^{9}$, Hiroshi Segawa ${ }^{9}$, Hairen Tan ${ }^{10}$, Qunwei Tang ${ }^{11}$, Junfeng Fang ${ }^{12}$, Yaowen $\mathrm{Li}^{13}$, \\ Liming Ding ${ }^{14}$, Zhijun Ning ${ }^{15}$, Yabing $\mathrm{Qi}^{16}$, Yiqiang Zhang ${ }^{17}$, Liyuan Han ${ }^{1,9} \bowtie$
}

\section{HIGHLIGHTS}

- Recent progress of efficiency and long-term stability for perovskite solar cells, and the development of perovskite-based tandem solar cells are described.

- The progress of lead-free perovskite solar cells and their potential for industrial production are discussed in detail.

- The current status, ongoing challenges, and the future outlooks of perovskite solar cells are highlighted.

ABSTRACT Perovskite solar cells (PSCs) emerging as a promising photovoltaic technology with high efficiency and low manufacturing cost have attracted the attention from all over the world. Both the efficiency and stability of PSCs have increased steadily in recent years, and the research on reducing lead leakage and developing eco-friendly lead-free perovskites pushes forward the commercialization of PSCs step by step. This review summarizes the main progress of PSCs in 2020 and 2021 from the aspects of efficiency, stability, perovskite-based tandem devices, and lead-free PSCs. Moreover, a brief discussion on the development of PSC modules and its challenges toward practical application is provided.

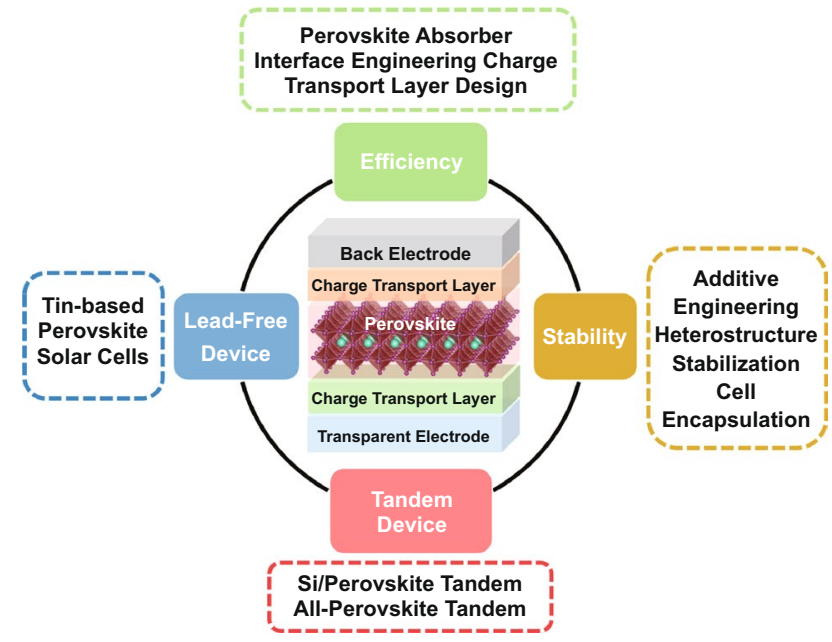

KEYWORDS Perovskite solar cells; Stability; Solar module; Perovskite-based tandem devices; Lead-free perovskite

Liyuan Han, han.liyuan@sjtu.edu.cn

${ }^{1}$ State Key Laboratory of Metal Matrix Composites, School of Material Science and Engineering, Shanghai Jiao Tong University, Shanghai 200240, People's Republic of China

2 Key Laboratory for Advanced Materials and Feringa Nobel Prize Scientist Joint Research Centre, Shanghai Key Laboratory of Functional Materials Chemistry, Joint International Research Laboratory of Precision Chemistry and Molecular Engineering, School of Chemistry and Molecular Engineering, East China University of Science and Technology, Meilong Road 130, Shanghai 200237, People's Republic of China

3 Wuhan National Laboratory for Optoelectronics, Huazhong University of Science and Technology, Luoyu Road 1037, Wuhan 430074, People's Republic of China

${ }^{4}$ College of Materials Science and Engineering, Nanjing University of Science and Technology, Nanjing 210094, People's Republic of China

5 Beijing Key Laboratory of Novel Thin-Film Solar Cells and State Key Laboratory of Alternate Electrical Power System With Renewable Energy Sources, North China Electric Power University, Beijing 102206, People's Republic of China

6 Department of Microelectronic Science and Engineering, Ningbo University, Zhejiang 315211, People's Republic of China 


\section{Introduction}

Perovskite solar cells (PSCs) have become a promising thinfilm photovoltaic (PV) technology due to the high lightabsorption coefficient, long carrier diffusion length, and solution processibility of metal halide perovskite materials [1-5]. Currently, the highest power conversion efficiency (PCE) of PSCs has reached 25.5\% [6], exceeding the record efficiency of copper indium gallium selenium (CIGS) solar cells and approaching that of crystalline-Si solar cells. Moreover, the scale-up deposition techniques for perovskites and charge transport layers promote the development of largearea perovskite solar modules, including doctor-blade coating, slot-die coating, screen printing, and spray deposition strategies. Recently, a certified efficiency of about $18 \%$ has been reported for an over $800 \mathrm{~cm}^{2}$ PSC sub-module [6], indicating large potential for practical use. On the other hand, perovskite-based tandem solar cells with a theoretical PCE beyond the limit of single-junction PSCs also make a huge progress owing to the reduction of defect density and increase of carrier diffusion length in both wide-bandgap and narrow-bandgap perovskite absorbers [7-9]. By now, the highest certified efficiency of the perovskite-based tandems has increased to over $29 \%$ [10].

Besides the efficiency progress, the long-term stability of PSCs against damp, light, and heat also improved significantly in recent years, which could be attributed to the construction of diffusion barrier against ion migration, additive engineering, design of chemically inert carbon-based electrodes, and development of cell encapsulation technique to reduce the lead leakage from a broken PSC module [11-18]. It was demonstrated that the printable PSCs had passed the most popular international standards of IEC61215:2016 for mature PV technology [19].

With the continuous progress of PSCs toward commercialization, exploiting eco-friendly lead-free perovskite materials has also become a hot research topic in this field in view of the toxicity of $\mathrm{Pb}$ element in $\mathrm{Pb}$-containing PSCs giving rise to the concern of environmental pollution [20-23]. So far, the highest certified efficiency of lead-free PSCs has reached $11.22 \%$, enabled by minimizing the defect density in tin halide perovskite films via a template-growth deposition method [24].

In this review, we summarize the representative works on PSCs published by worldwide research groups in 2020-2021 from the aspects of efficiency, stability, perovskite-based tandem solar cells, and the development of lead-free PSCs. In addition, we point out the future challenges on realizing the commercialization of PSCs, and then give a brief outlook on the possible research topics at the next stage.

\section{Efficiency}

PSCs are usually composed of perovskite absorbers, charge transport layers and counter electrodes. The energy loss in the bulk and interface of perovskites layers, and the charge extraction and transportation process in device play a critical role in determining the efficiency of PSCs. Therefore, improving the crystal quality of perovskite films, suppressing the non-radiative recombination at interface, and rational

\footnotetext{
7 College of Chemical Engineering, Jiangsu Key Lab of Biomass-Based Green Fuels and Chemicals, Nanjing Forestry University, Nanjing 210037, People's Republic of China

8 College of Chemistry and Molecular Engineering, Qingdao University of Science and Technology, Qingdao 266042, People's Republic of China

9 Special Division of Environmental and Energy Science, Komaba Organization for Educational Excellence (KOMEX), College of Arts and Sciences, University of Tokyo, Tokyo 153-8902, Japan

${ }^{10}$ National Laboratory of Solid State Microstructures, Jiangsu Key Laboratory of Artificial Functional Materials, College of Engineering and Applied Sciences, Nanjing University, Nanjing 210093, People's Republic of China

11 College of Information Science and Technology, Jinan University, Guangzhou 510632, People's Republic of China

12 School of Physics and Electronic Science, Engineering Research Center of Nanophotonics and Advanced Instrument, Ministry of Education, East China Normal University, Shanghai 200062, People's Republic of China

${ }^{13}$ Laboratory of Advanced Optoelectronic Materials, College of Chemistry, Chemical Engineering and Materials Science, Soochow University, Suzhou 215123, People's Republic of China

${ }^{14}$ Center for Excellence in Nanoscience, Key Laboratory of Nanosystem and Hierarchical Fabrication, National Center for Nanoscience and Technology, Beijing 100190, People's Republic of China

15 School of Physical Science and Technology, ShanghaiTech University, 100 Haike Road, Shanghai 201210, People's Republic of China

${ }^{16}$ Energy Materials and Surface Sciences Unit (EMSSU), Okinawa Institute of Science and Technology Graduate University (OIST), Okinawa 904-0495, Japan

${ }^{17}$ School of Materials Science and Engineering, Henan Institute of Advanced Technology, Zhengzhou University, Zhengzhou 450001, People's Republic of China
} 
design of the charge transport layers are expected to further improve the device efficiency.

\subsection{Perovskite Absorbers}

In 2020-2021, the research activities on perovskite layer mainly focus on stabilizing the formamidinium lead iodide $\left(\mathrm{FAPbI}_{3}\right)$ perovskite phase with wide absorption range and long carrier lifetime to boost the short-circuit current density $\left(J_{\mathrm{SC}}\right)$ and open-circuit voltage $\left(V_{\mathrm{OC}}\right)$ of PSCs $[25,26]$. Besides, exploiting the single-crystal device with very low defect density has also been reported for realizing highperformance PSCs.

To maximize the photon-absorption at the UV-vis region, Kim et al. used the inherent bandgap $(1.47 \mathrm{eV})$ of $\alpha$-phase $\mathrm{FAPbI}_{3}$ to increase the photocurrent of PSCs [27]. They replaced the FA cations by a trace amount $(0.03$ molar fraction) of cesium (Cs) and methylenediammonium (MDA) cations to stabilize the $\alpha$-phase $\mathrm{FAPbI}_{3}$ without changing its inherent bandgap, but improved the UV-vis absorption of perovskite layer. These effects contribute to a high $J_{\mathrm{SC}}$ of $26.23 \mathrm{~mA} \mathrm{~cm}^{-2}$ for PSCs, very close to the theoretical current limit of about $27 \mathrm{~mA} \mathrm{~cm}{ }^{-2}$ for $1.47 \mathrm{eV}$-bandgap semiconductors [7]. Moreover, Fig. 1a shows that co-doping of $0.03 \mathrm{~mol} \mathrm{Cs}$ and MDA cations also relaxes the lattice strain of $\mathrm{FAPbI}_{3}$ by over $70 \%$ compared to the sample only treated with MDA cation reported in their previous study [28], which prolongs the carrier recombination lifetime and enables a $V_{\mathrm{OC}}$ increase of $30 \mathrm{mV}$ (Fig. 1b), leading to a high certified PCE reaching $24.4 \%$.

Besides the cation-doping strategies, substitution of $\mathrm{X}$-site halide anions could also significantly affect the optoelectronic properties of $\mathrm{FAPbI}_{3}$ perovskites. Jeong et al. introduced an anion engineering strategy that employs the pseudo-halide anion formate $\left(\mathrm{HCOO}^{-}\right)$to fill the halide
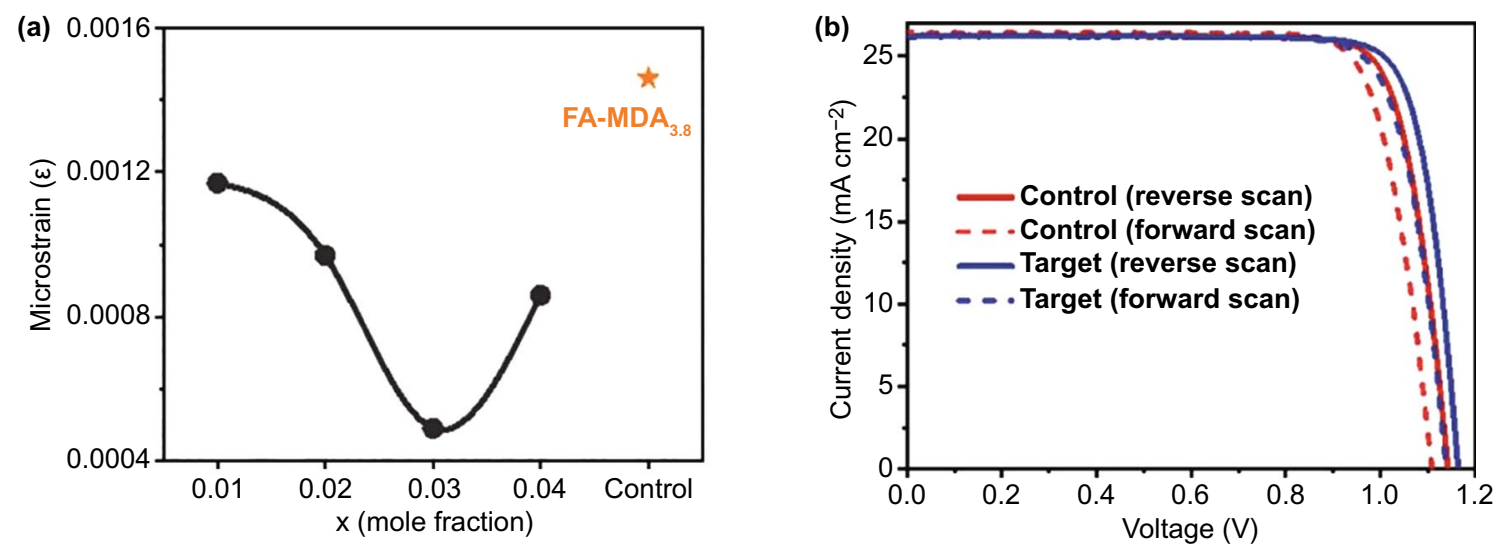

(c)

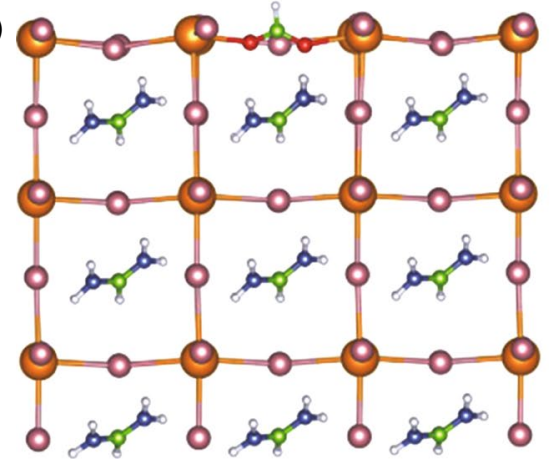

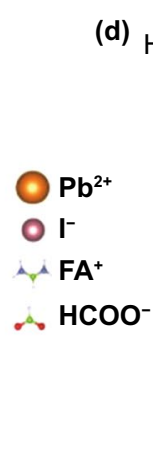

(d)

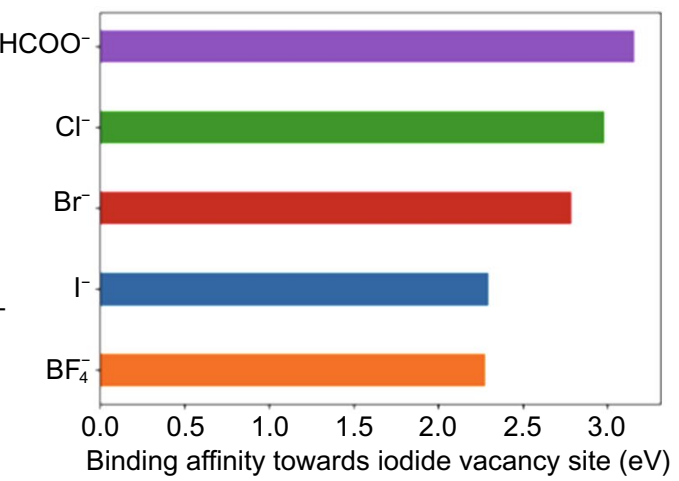

Fig. 1 a Residual strain calculated in $\mathrm{FAPbI}_{3}$ perovskite films doped with different molar ratio (x) of Cs and MDA cations, and the control sample treated with 3.8 mol\% MDA. b The best $J-V$ curves of the control device and the PSCs treated with Cs and MDA. Reproduced with permission from Ref. [27] Copyright 2020 AAAS. c Calculated crystal structure demonstrating the passivation of $\mathrm{I}^{-}$vacancy at $\mathrm{FAPbI}_{3}$ surface by a $\mathrm{HCOO}^{-}$anion. All chemical compounds are shown in ball-and-stick style. $\mathbf{d}$ The relative binding energy of different anions at the site of $\mathrm{I}^{-}$ vacancy at $\mathrm{FAPbI}_{3}$ surface. Reproduced with permission from Ref. [29] Copyright 2021 Nature Publishing Group 
vacancy defects located at grain boundaries and surface of perovskite films [29] and to enhance the crystallinity of $\mathrm{FAPbI}_{3}$. It is found that the doping of $2 \%$ formate anions could enlarge the grain size to about $2 \mu \mathrm{m}$, increasing the crystal orientation along (100) and (200) directions that are better for carrier transport, and suppressing the formation of non-photoactive $\delta-\mathrm{FAPbI}_{3}$ phase. Moreover, the theoretical calculation revealed that formate anions had a larger binding affinity toward iodide vacancy sites compared to other anions like $\mathrm{Cl}^{-}, \mathrm{Br}^{-}$, and $\mathrm{BF}_{4}{ }^{-}$owing to the fact that every carboxylate group can form two $\mathrm{Pb}-\mathrm{O}$ coordination bonds with the lead cations (Fig. 1c, d). As a result, the $\mathrm{FAPbI}_{3}$-based PSCs with pseudo-halide treatment attained a record PCE of $25.6 \%$ (certified $25.2 \%$ ) and a $V_{\mathrm{OC}}$ of $1.19 \mathrm{~V}$.

Another representative work for the improvement in perovskite layers is the fabrication of uniaxial-oriented perovskite layer with millimeter-sized grains via a methylamine gas-assisted crystallization method [30]. In this study, the methylammonium lead iodide $\left(\mathrm{MAPbI}_{3}\right)$ perovskite film was formed by controlling the evaporation rate of MA gas molecules from liquid intermediate phase of $\mathrm{MAPbI}_{3} \cdot \mathrm{xMA}$ in a closed system. The results demonstrated that a slow release rate of MA molecules from the liquid intermediate phase significantly reduced the supersaturation, leading to a low nucleation density that provided more time for growing large $\mathrm{MAPbI}_{3}$ grains during thermal annealing process. Consequently, the perovskite grains with about $1 \mathrm{~mm}-$ size can be obtained, resulting in a very low trap density of $9.7 \times 10^{13} \mathrm{~cm}^{-3}$, approaching that of the $\mathrm{MAPbI}_{3}$ single crystal [31], and the PSCs with millimeter-sized $\mathrm{MAPbI}_{3}$ perovskite grains exhibited a promising PCE of $21.36 \%$.

\subsection{Interface Engineering}

The interfacial properties between perovskite and charge transport layers play an important role in the charge recombination mechanism of PSCs, which greatly influence the $V_{\mathrm{OC}}$ and fill factor (FF) of the solar cells [32-34]. Therefore, many methods have been developed to reduce the non-radiative recombination loss at the interface and optimize the series resistance of the passivation layers for efficiency improvement.

To suppress carrier recombination at perovskite surface and grain boundaries, Zheng et al. used a trace amount of surface-anchoring alkylamine ligands (AALs) with different chain lengths for the modification of perovskite surface
[35]. They found that the oleylamine ligand with a long alkyl chain showed a more obvious passivation effect for improving carrier recombination lifetime and the $V_{\mathrm{OC}}$ of PSCs (Fig. 2a), as compared to other organic AALs with shorter alkyl chains, which could be attributed to the electron-tunneling effect in the insulating AALs layers. Such a tunneling effect allows the movement of electrons from perovskite conduction band to the lowest unoccupied molecular orbital (LUMO) of the fullerene- $\mathrm{C}_{60}$ layer, but effectively blocks the hole injection from valence band to the highest occupied molecular orbital (LUMO) of $\mathrm{C}_{60}$ (Fig. 2b). This passivation technique based on the insulating tunneling layer is also widely applied in silicon solar cells [36]. Based on this oleylamine ligand-anchoring strategy, the invertedstructure PSCs achieved a strong $V_{\mathrm{OC}}$ improvement up to $110 \mathrm{mV}$, resulting in a record certified efficiency of $22.3 \%$.

Although the additional passivation layer is beneficial for the $V_{\mathrm{OC}}$ improvement of PSCs, it may undesirably increase the series resistance of devices and thus leads to the loss of FF. This detrimental effect will become more obvious in the case of large-area devices. In order to balance the improvement of $V_{\mathrm{OC}}$ and FF, Peng et al. designed a nanoscale localized contact at the polymer-passivated perovskite/ $/ \mathrm{TiO}_{2}$ interface to realize a higher FF for $1 \mathrm{~cm}^{2}$ PSCs [37]. They deposited a nanopatterned electron-selective $\mathrm{TiO}_{2}$ layer via atomic-layer deposition (ALD) to form nanorod-like charge transport channels through the passivated interface (the device structure is shown in Fig. 2c), which provided both effective contact passivation and excellent charge extraction ability for reducing the device series resistance. As a result, they achieved a high FF of $83.9 \%$ and a high $V_{\text {OC }}$ of $1.20 \mathrm{~V}$ by employing an ALD $\mathrm{TiO}_{2}$ nanopattern with $300 \mathrm{~nm}$ spacing at the polymer passivation interface (Fig. 2d), resulting in a record certified PCE of $21.6 \%$ for an over $1 \mathrm{~cm}^{2}$ cell.

\subsection{Charge Transport Layer Design}

Rational design of the charge transport layer also boosts the PV parameters of PSCs significantly. Jeong et al. designed two fluorinated isomeric analogs of the well-known hole transport molecule spiro-OMeTAD to modify the energylevel alignment, hydrophobicity, and hole extraction ability in PSCs [38]. They found that the fluorinated group at the meta-position on the benzene ring (spiro-mF) could 
(a)

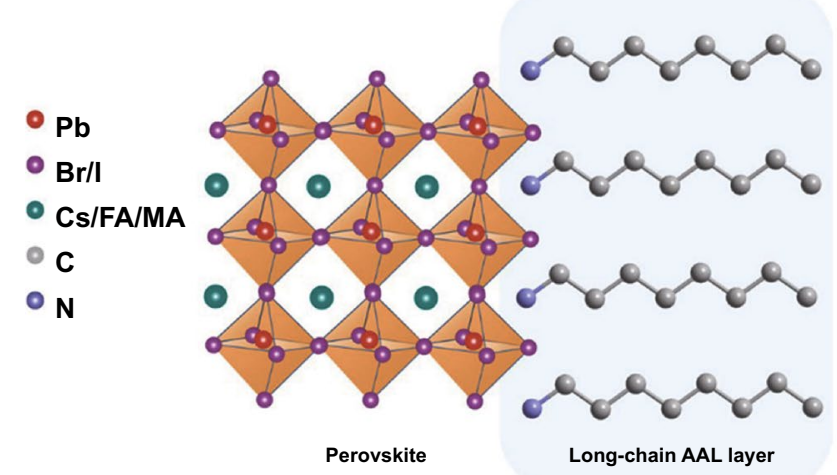

(b)

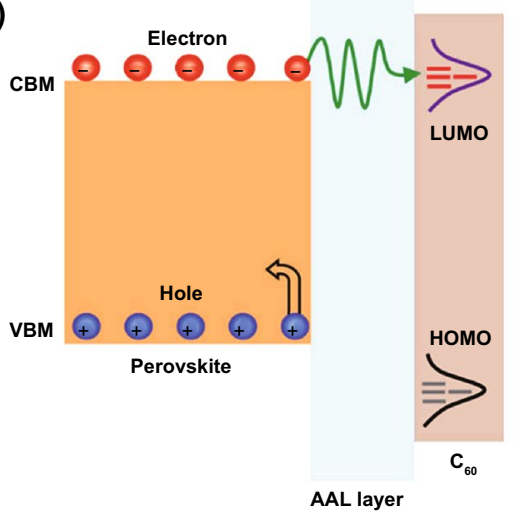

(c)
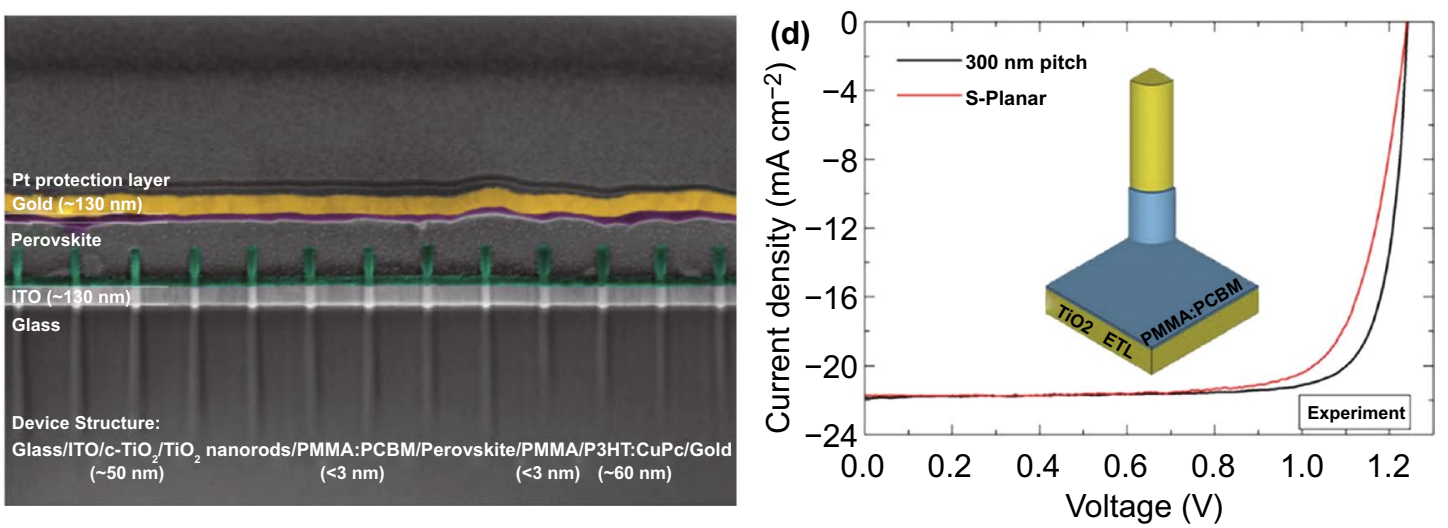

Fig. 2 a Illustration of the long-chain AALs assembled on perovskite film surface. $\mathbf{b}$ The electron-tunneling and hole-blocking effects induced by AAL layer at the perovskite/ $\mathrm{C}_{60}$ interface, $\mathrm{VBM}$ and $\mathrm{CBM}$ represent the valence band maximum and conduction band minimum of perovskite semiconductor, respectively. Reproduced with permission from Ref. [35] Copyright 2020 Nature Publishing Group. c Cross-sectional SEM image of the PSCs designed with $\mathrm{TiO}_{2}$ nanorod pattern at the bottom surface of perovskite layer. d Experimental $J$ - $V$ curves of the PSCs based on planar (reference cell) and the $\mathrm{TiO}_{2}$ nanopattern structure, $300 \mathrm{~nm}$ pitch represents the $\mathrm{TiO}_{2}$ nanorods with a spacing of $300 \mathrm{~nm}$ in devices, the inset shows that about $30 \%$ of the nanorod is coated with insulating passivation layer. Reproduced with permission from Ref. [37] Copyright 2020 AAAS

lower the HOMO position from -4.97 to $-5.19 \mathrm{eV}$ and thus provided a more suitable energy-level alignment with the valence band maximum (VBM) of $\mathrm{FAPbI}_{3}$ perovskite absorber $(-5.40 \mathrm{eV})$ to reduce the interfacial energy loss, resulting in a $V_{\mathrm{OC}}$ improvement of the device. Moreover, the F atoms could induce a denser solid-state molecule packing through non-covalent intramolecular interaction, which further improves the electronic contact between spiro- $\mathrm{mF}$ and perovskite surface, producing better transport and extraction ability of holes, resulting in a slightly increase of $J_{\mathrm{SC}}$ and FF. These effects boost the efficiency from $23.44 \%$ to 24.84\% for PSCs.

On the other side, chemical bath deposition was applied to produce a high-quality electron-selective $\mathrm{SnO}_{2}$ layer for limiting excess charge carrier recombination in PSCs [39].
Unlike the conventional deposition using $\mathrm{SnO}_{\mathrm{x}}$ nanoparticle dispersion, chemical bath deposition enables the uniform and complete coverage of $\mathrm{SnO}_{2}$ film on the substrate via a rational control of the reaction time and $\mathrm{pH}$ value of the $\mathrm{SnCl}_{2}$ precursor solution. As can be seen in Fig. 3, at the stage $\mathrm{A}-\mathrm{i}$, with a low $\mathrm{pH}$ value and short reaction time, the as-deposited $\mathrm{SnO}_{2}$ film showed many pinholes. After increasing the $\mathrm{pH}$ value to stage $\mathrm{A}$-ii, a low O-vacancy $\mathrm{SnO}_{2}$ film (targeted sample) with ideal film coverage, thickness, and chemical composition could be achieved. When further increasing the $\mathrm{pH}$ and reaction time to stages A-iii and $\mathrm{B}$, the density of $\mathrm{O}$-vacancy increased significantly and the additional $\mathrm{Sn}_{6} \mathrm{O}_{4}(\mathrm{OH})_{4}$ and $\mathrm{SnO}$ phases were present, lowering the electron transport ability and inducing charge recombination in $\mathrm{SnO}_{2}$ films. By further optimizing the $\mathrm{Br}$ 


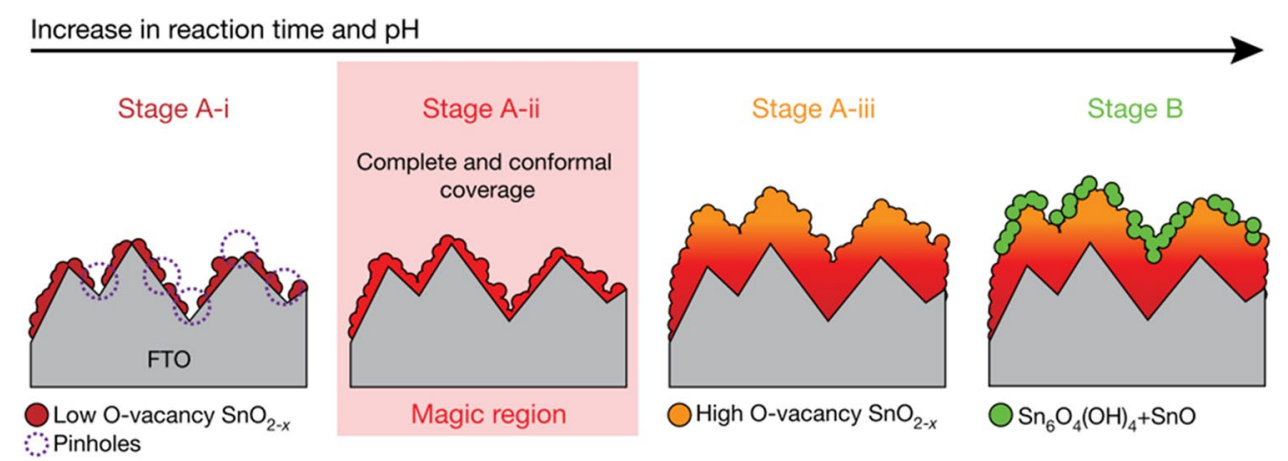

Fig. 3 Schematic illustration of four major stages at different reaction time and $\mathrm{pH}$ value of the chemical bath deposition for $\mathrm{SnO}_{2}$, the magic

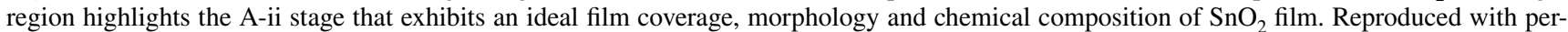
mission from Ref. [39] Copyright 2021 Nature Publishing Group

concentration in perovskite films, a certified PCE of $25.2 \%$ was achieved, corresponding to $80.5 \%$ of the theoretical efficiency limit.

\section{Stability}

Stability issues are the major bottleneck for the commercialization of PSCs. The chemical components of metal halide perovskite are bonded through weak interactions such as ionic interaction, hydrogen bonding, and van der Waals forces, which results in the soft-material nature of perovskite semiconductors. Irreversible decomposition of organic species and the ion migration occur easily in PSCs under moisture penetration, continuous light soaking, thermal stress, and external electric field [11, 40-42], which cause damage to both the perovskite and charge transport layers. On the other hand, the leakage of $\mathrm{Pb}$ during long-term operation of PSCs under bad weather conditions could further cause environmental and public health risk, which should also be considered as an important stability issue to be addressed for future commercialization. In this part, we discuss the recent research progress on PSC stability from the viewpoint of additive engineering, heterostructure stabilization, and the cell encapsulation technology for reducing lead leakage.

\subsection{Additive Engineering}

Introducing the additive molecules that could form extra chemical interactions with the volatile components is an effective way to suppress ion migration and irreversible decomposition of perovskite films. Mei et al. [19] introduced bifunctional 5-ammoniumvaleric acid iodide (5-AVAI) to inhibit the methylammonium iodide (MAI) loss on the surface and the crystal reconstruction of $\mathrm{MAPbI}_{3}$ grains. 5-AVAI could form hydrogen bonds with the iodine ions through amino groups $\left(-\mathrm{NH}_{3}{ }^{+}\right)$, while their carboxyl groups (-COOH) form a strong hydrogen bond with another 5-AVAI in the adjacent grains, which results in a cross-linking of the perovskite surface and interface and a large enhancement of the thermal stability. Besides, non-volatile 5-AVAI at the interfaces limits the volatilization of organic components, which inhibits the forward process of decomposition reaction of $\mathrm{MAPbI}_{3}$. It can also stabilize the metal oxide matrix $\left(\mathrm{ZrO}_{2}\right.$ and $\left.\mathrm{TiO}_{2}\right)$ via the anchoring effect between - $\mathrm{COOH}$ and metal cations, thus restricting ion migration of $\mathrm{MA}^{+}$and $\mathrm{I}^{-}$at the grain boundaries and the interfaces. After encapsulation with a hot-melt polymer film, the printable PSCs treated with 5-AVAI successfully passed the main items of IEC61215:2016 qualification tests, especially working for more than $9000 \mathrm{~h}$ at a maximum power point of $55 \pm 5{ }^{\circ} \mathrm{C}$ without obvious decay.

Furthermore, an organic ionic salt called 1-butyl-1-methylpiperidinium tetrafluoroborate $\left([\mathrm{BMP}]^{+}\left[\mathrm{BF}_{4}\right]^{-}\right)$was developed to suppress the light-induced phase segregation and degradation in thermally stable CsFA-based lead-halide perovskites [43]. The results indicated that small amounts of $[\mathrm{BMP}]^{+}\left[\mathrm{BF}_{4}\right]^{-}$additives could penetrate the entire volume of perovskite film and passivate both the bulk and surface defect states via ionic interaction, leading to a large photovoltage enhancement for the PSCs. During the aging process, $[\mathrm{BMP}]^{+}\left[\mathrm{BF}_{4}\right]^{-}$prevented the segregation of $\mathrm{Br}$-rich $\mathrm{FAPbBr}_{3}$ phase in mixed-halide perovskite film and suppress the corrosion of silver electrode (one of the key reasons for device degradation) induced by $\mathrm{I}_{2}$ generation under 
light and heat. $I_{2}$ is mainly formed by the combination of interstitial $\mathrm{I}^{-}$and holes, or two neutral iodine atoms [44]. In the presence of $[\mathrm{BMP}]^{+}\left[\mathrm{BF}_{4}\right]^{-}$, the ion diffusion channels at the grain surface were effectively reduced, resulting in the inhibition of $\mathrm{I}_{2}$ formation. Consequently, under full-spectrum simulated sunlight in ambient atmosphere, the unencapsulated and encapsulated PSCs retained 80\% and $95 \%$ of their post-burn-in efficiencies for $1010 \mathrm{~h}\left(60^{\circ} \mathrm{C}\right)$ and $1200 \mathrm{~h}\left(85^{\circ} \mathrm{C}\right)$, respectively.

Other additives such as lead chloride, organic dye molecules, phosphorus-based Lewis acid, and cyano derivatives have been reported to suppress ion migration and passivate surface ionic defects in perovskite film, resulting in the improvement of long-term stability [45-47].

\subsection{Heterostructure Stabilization}

The heterostructures formed between perovskite and charge transport layers play a significant role in the long-term stability of the whole device, so much attention has been paid to stabilizing the interface of each functional layer in PSCs. Liu et al. employed a holistic strategy to stabilize the front and back interface of perovskite layer under operational condition [48].They used ethylenediaminetetraacetic acid dipotassium salt (EDTAK) to treat the electron-selective $\mathrm{SnO}_{2}$ surface for stabilization of the front heterostructure (Fig. 4a). The Lewis acid-base reaction between the alkylamine group of EDTAK and $\mathrm{Pb}^{2+}$ cations efficiently passivated the vacancy defects in the perovskite film. Besides, incorporation of EDTAK also shifted the conduction band minimum (CBM) of $\mathrm{SnO}_{2}$ from -3.69 to $-3.95 \mathrm{eV}$, resulting in a better energy-level alignment for charge extraction. The perovskite/ spiro-OMeTAD heterostructure was stabilized by the surface ethylammonium iodide (EAI) modification to form an EAMA-based perovskite capping layer with better ambient stability. Regarding the spiro-OMeTAD/electrode interface, a small amount of P3HT was incorporated to inhibit the degradation caused by inward migration of gold into the

(a)
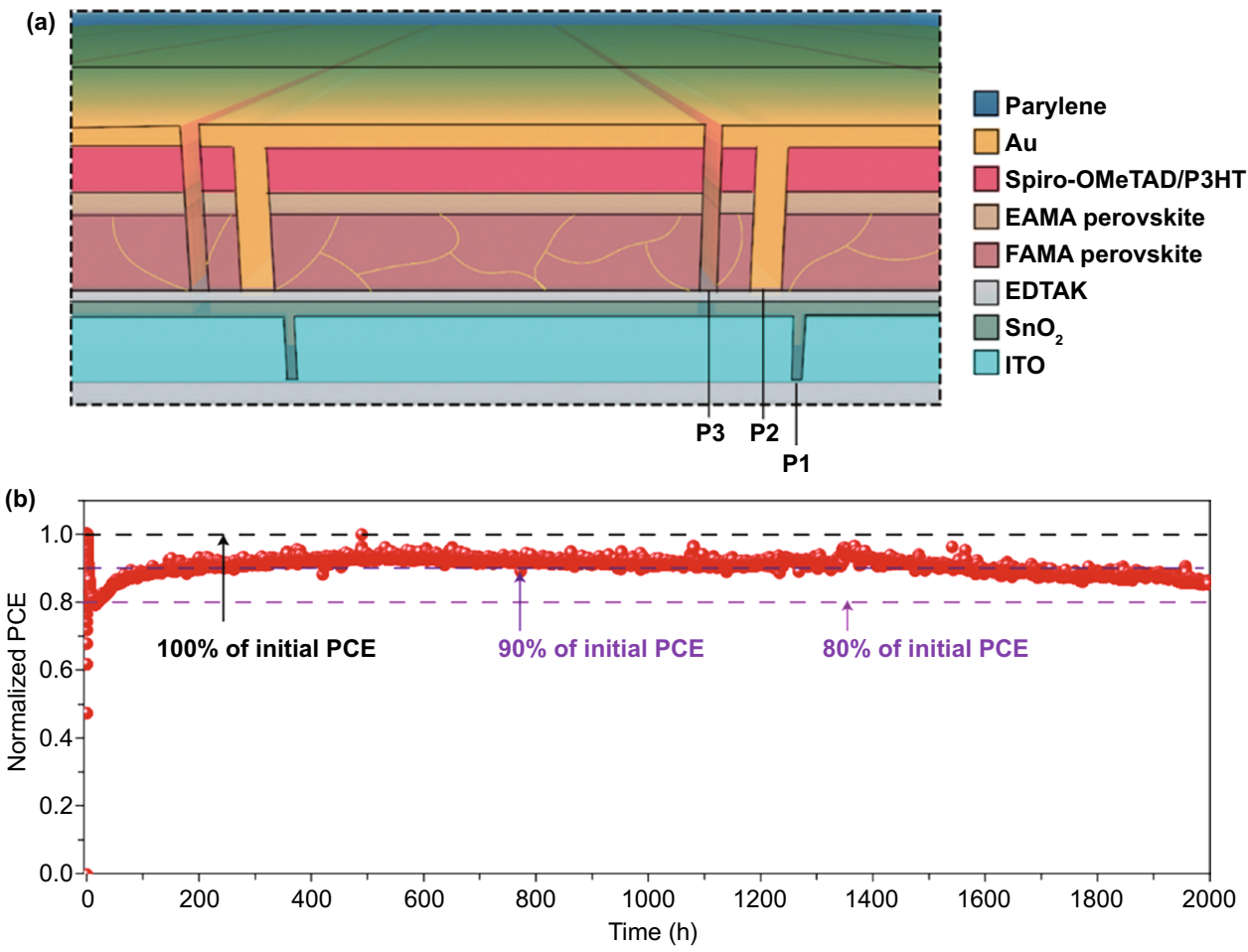

Fig. 4 a The holistic method containing four treatments for the functional layers and their interfaces in device, including the use of EDTAK to modify $\mathrm{SnO}_{2}$ layer, the use of ethylammonium iodide (EAI) to form EAMA-based perovskite atop the FAMA-based perovskite absorber, doping $\mathrm{P} 3 \mathrm{HT}$ into the spiro-OMeTAD layer, and using parylene to encapsulate the PSC module. b The operational stability of parylene-encapsulated solar module with a structure of $\mathrm{SnO}_{2}$-EDTAK/perovskite/spiro-OMeTAD-P3HT/Au measured under continuous 1-sun illumination. Reproduced with permission from Ref. [47] Copyright 2020 Nature Publishing Group 
perovskite layer and also enhance the moisture stability of spiro-OMeTAD. By employing such a holistic stabilization approach, the PSC modules without encapsulation achieved an efficiency of $16.6 \%$ with a designated area of $22.4 \mathrm{~cm}^{2}$, and the encapsulated solar modules with parylene-coated cover glass retained approximately $86 \%$ of the initial performance after continuous operation for $2000 \mathrm{~h}$ under AM1.5G light illumination (Fig. 4b).

Our previous work employed CsFA-based perovskite with high thermal stability as the absorber to construct a scalable integrated heterostructure for PSC modules [49]. Under the operational condition, the iodide ions can easily migrate from perovskite to the hole transport layer (HTL) and change its semiconducting properties from p-type to n-type, causing deterioration of hole extraction in PSCs. To stabilize this heterostructure, a scalable bridge-jointed graphene oxide (BJ-GO) layer was deposited on the perovskite surface to block iodide migration. During this process, 3-aminopropyl triethoxysilane (APTES) was used to connect the small-size GO nanosheets by forming C-N covalent bonds with GO, resulting in a full-coverage hydrophobic blocking layer on perovskite surface for inhibiting ion migration, passivating surface under-coordinated $\mathrm{Pb}^{2+}$, and protecting perovskite layer from the damage of water in ambient environment. By employing the BJ-GO layer in the PSCs with undoped HTL, we achieved a high PCE of $16.21 \%$ for a $36 \mathrm{~cm}^{2}$ solar module. The encapsulated module retained over $91 \%$ of its initial efficiency after the damp heat test at $85{ }^{\circ} \mathrm{C}$ and $85 \%$ relative humidity for $1000 \mathrm{~h}$, while maintaining $90 \%$ of the initial value for $1000 \mathrm{~h}$ under standard operational condition at $60{ }^{\circ} \mathrm{C}$.

\subsection{Cell Encapsulation}

In 2020-2021, researches on PSC encapsulation mainly focused on reducing the $\mathrm{Pb}$ leakage from a broken device. During the long-term operation process, $\mathrm{Pb}^{2+}$ showing weak chemical interaction with other perovskite components could also escape from perovskite layer after water penetration, resulting in additional environmental problems. For a typical lead perovskite absorber with a thickness of $550 \mathrm{~nm}$, the unit-area lead concentration is estimated to be about $0.75 \mathrm{~g} \mathrm{~m}^{-2}$, which is more than 100 times higher than that of the commonly used $\mathrm{Pb}$-containing paints $\left(0.007 \mathrm{~g} \mathrm{~m}^{-2}\right)$ [50].
The physical encapsulation with $\mathrm{Pb}^{2+}$-absorption materials can effectively suppress the irreversible outflow of $\mathrm{Pb}$ from damaged devices into the underground water or soils at severe weather conditions [51]. Li et al. deposited the leadabsorbing materials on both front and back sides of PSCs to reduce the leakage of lead [23]. On the front glass side, they used a transparent $\mathrm{Pb}$-absorbing molecule with phosphonic acid groups that showed a large binding energy with $\mathrm{Pb}^{2+}$ cations to absorb lead in water when water seeps into the device. On the back side, they coated a polymer film mixed with lead-coordination agents between the metal electrode and the encapsulation layer. As a consequence, the leadabsorption effects on both sides of PSCs could sequestrate more than $96 \%$ of lead leakage in water induced by severe device damage while retain the structural integrity of solar cells.

Furthermore, a low-cost and chemically robust cationexchange resin (CER)-based method was developed to prevent lead leakage from broken PSC modules [52]. The results showed that CER exhibited both high adsorption capacity and high adsorption rate of $\mathrm{Pb}^{2+}$ in water due to the high binding energy between sulfonate-terminated groups and the common divalent metal ions like $\mathrm{Pb}^{2+}, \mathrm{Ca}^{2+}$, and $\mathrm{Mg}^{2+}$ in the mesoporous polymer matrix (Fig. 5a). Additionally, mixing the CER with carbon electrode and integrating them on the front glass of PSC modules have a negligible detrimental impact on the device PCE while reduces lead leakage from mini-modules in water by 62 -fold to $14.3 \mathrm{ppb}$ as compared to that of the device without CER (Fig. 5b). The theoretical calculation indicates that the CER treatment could further reduce the lead leakage of large-area perovskite solar panels to lower than $7.0 \mathrm{ppb}$ even under the worst condition that all the PSC sub-modules are broken.

\section{Perovskite-Based Tandem Solar Cells}

The tunable bandgap of $\mathrm{ABX}_{3}$ perovskite absorber from $1.2 \mathrm{eV}$ to $3.0 \mathrm{eV}$ benefits the design of silicon-perovskite or perovskite-perovskite tandem devices [34] that enables the theoretical efficiency beyond the Shockley-Queisser limit of single-junction solar cells. For silicon-perovskite tandems, exploiting the ideal wide-bandgap perovskite materials $(1.6-1.7 \mathrm{eV})$ with suitable spectrum response matching the $1.1 \mathrm{eV}$-bandgap silicon absorber is key to obtaining high 
(a)

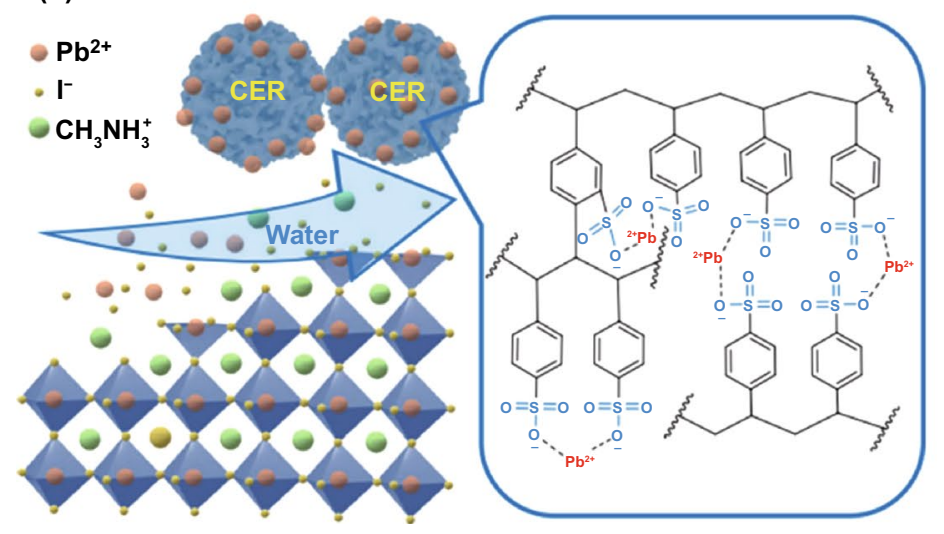

(b)

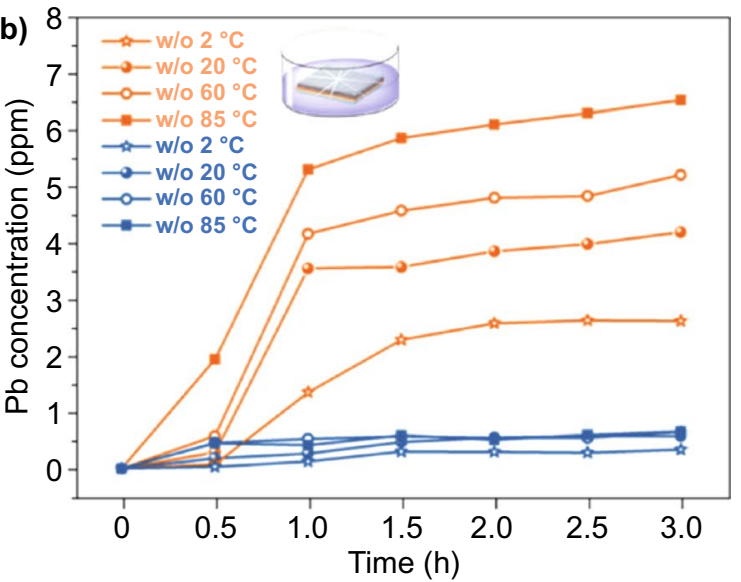

Fig. 5 a Illustration of how CERs prevent the lead leakage via strong ionic interaction between $\mathrm{Pb}^{2+}$ cations in broken perovskite films and the sulfonate groups. b Pb-soaking test results in water for the damaged perovskite solar modules without (yellow line) and with (blue line) the CER coating layer on glass side. Reproduced with permission from Ref. [50] Copyright 2020 Nature Publishing Group

efficiency [53]. By contrast, suppressing the generation of $\mathrm{Sn}^{4+}$ defects and increasing the carrier diffusion length in narrow-bandgap mixed $\mathrm{Sn}-\mathrm{Pb}$ perovskites (1.1-1.2 eV) are currently the major tasks for efficient all-perovskite tandem devices.

\subsection{Silicon-Perovskite Tandem Structure}

In the past two years, the efficiency of silicon-perovskite tandems boosted rapidly from about $25 \%$ to over $29 \%$ due to the reduced $V_{\mathrm{OC}}$ deficit of wide-bandgap perovskite top cell enabled by compositional engineering for a stable phase and the selection of charge transport layers [54-56]. Xu et al. [57] reported a triple-halide alloys containing chlorine, bromine, and iodine to tailor the carrier lifetime and suppress the light-induced phase segregation in wide-bandgap perovskite films. They found that direct incorporation of large amounts of $\mathrm{Cl}$ (more than 15\%) into the double-halide perovskite could produce a uniform halide distribution throughout the film with an ideal bandgap $(1.67 \mathrm{eV})$ matching the spectrum of the bottom 1.12-eV Si absorber. This effect increased the photocarrier mobility by two times and efficiently suppressed the phase separation that was previously found in most of the mixed-halide perovskite compositions $[58,59]$. By extending the double-halide to triple-halide components, a distinct efficiency enhancement for opaque single-junction PSCs from $18.15 \%$ to $20.42 \%$ was demonstrated, with a $V_{\mathrm{OC}}$ increasing over $100 \mathrm{mV}$. Moreover, they obtained a PCE of $27.04 \%$ in $1-\mathrm{cm}^{2}$ two-terminal monolithic tandems via integrating the optimized perovskite top cells with the silicon bottom cells.

Besides the modification of perovskite layer, a novel selfassembled, methyl-substituted carbazole monolayer (Me4PACz, the chemical structure is shown in Fig. 6a) was developed as the HTL to minimize the non-radiative recombination loss at the hole-selective contact in wide-bandgap PSCs [10], resulting in a record efficiency of $29.15 \%$ for Si-perovskite tandem devices. The carbazole unit provided fast hole extraction from the wide-bandgap perovskite layer and the methyl substitution was considered to passivate the defects on perovskite surface, minimizing the FF loss induced by series resistance (transport loss) and the interfacial non-radiative recombination in PSCs (Fig. 6b), as investigated by intensity-dependent absolute photoluminescence measurements. By using the Me-4PACz hole-selective layer, the FF of single-junction PSCs greatly increased from $79.8 \%$ (control cell based on PTAA) to $84.0 \%$, and the device $V_{\mathrm{OC}}$ also showed a large improvement from $1.19 \mathrm{~V}$ to $1.25 \mathrm{~V}$.

Textured crystalline-Si subcells have also been reported for Si-perovskite tandem devices to reduce the device reflectance and increase the photon usage rate. Hou et al. [60] directly deposited the solution-processed micrometer-thick perovskite top cell on a fully textured $\mathrm{Si}$-heterojunction bottom cell to fabricate tandem devices. They addressed the carrier-extraction issue in micrometer-thick perovskite layer by increasing the depletion width at the bases of silicon 
(a)

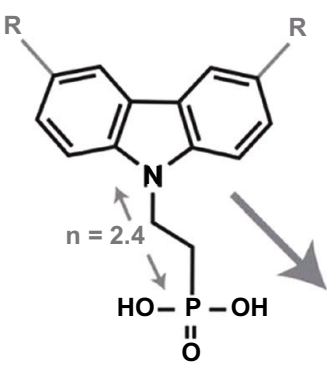

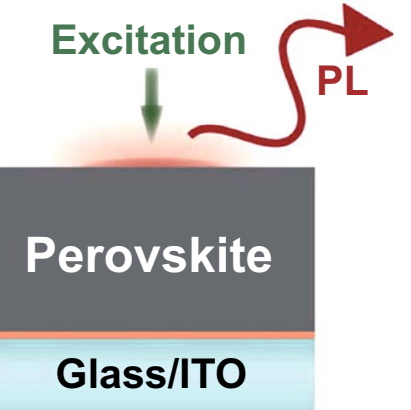

Excitation

(c)
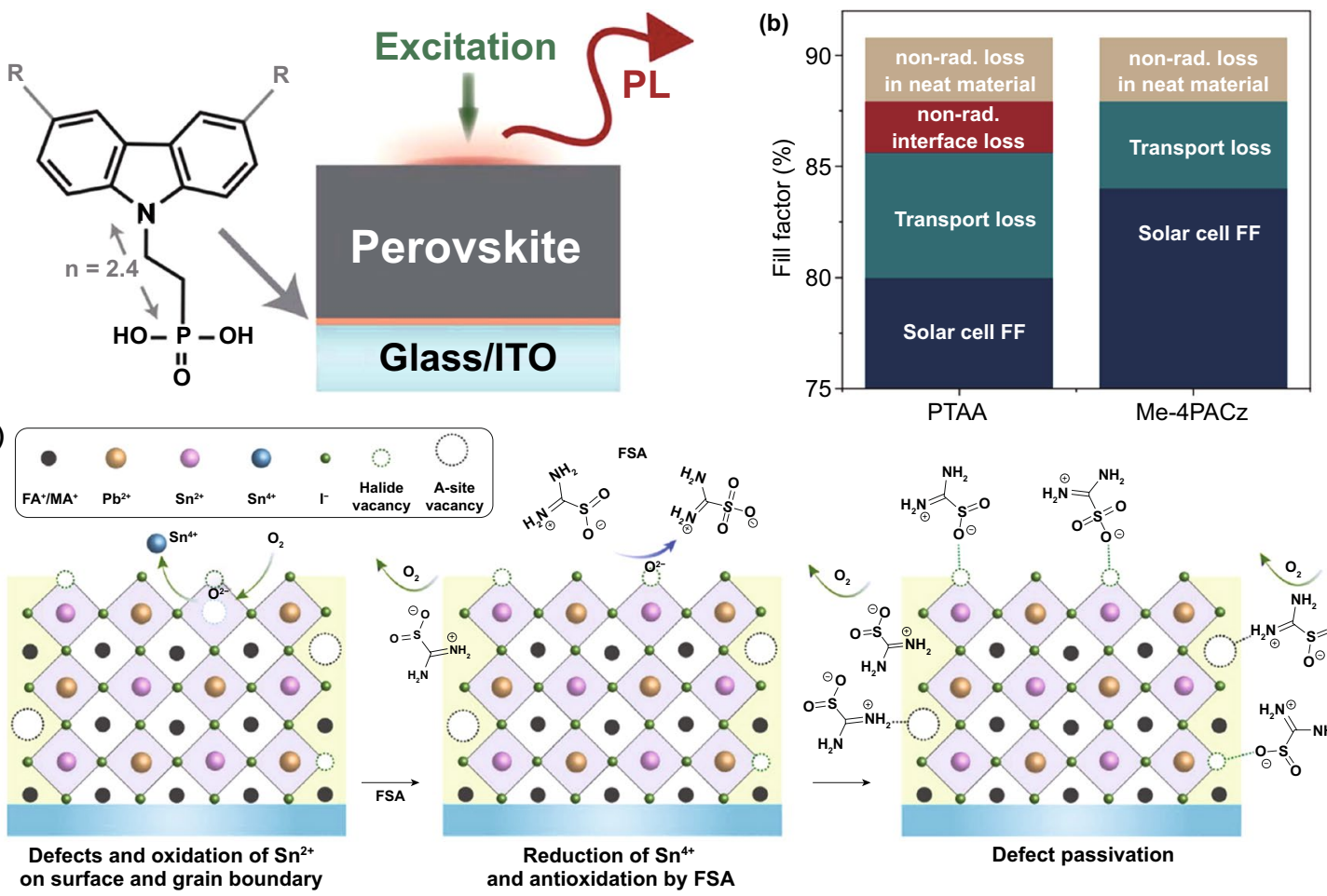

(d)

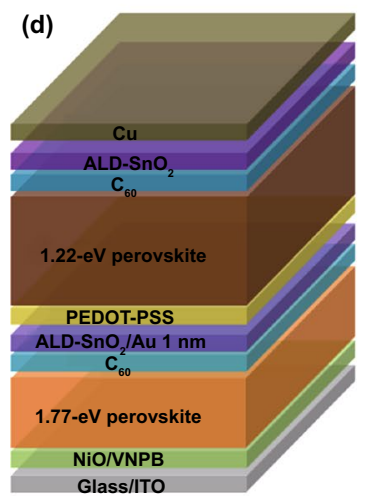

(e)

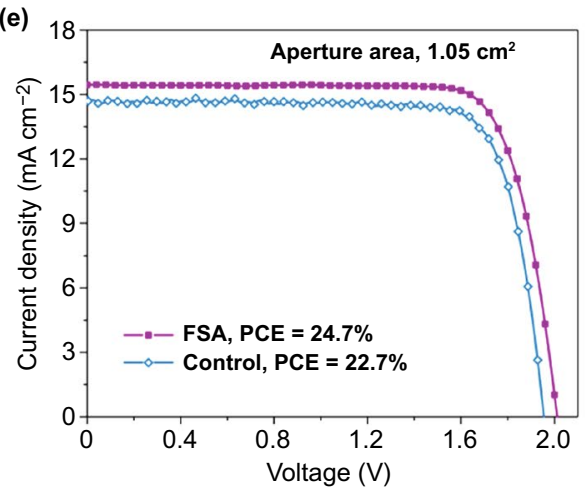

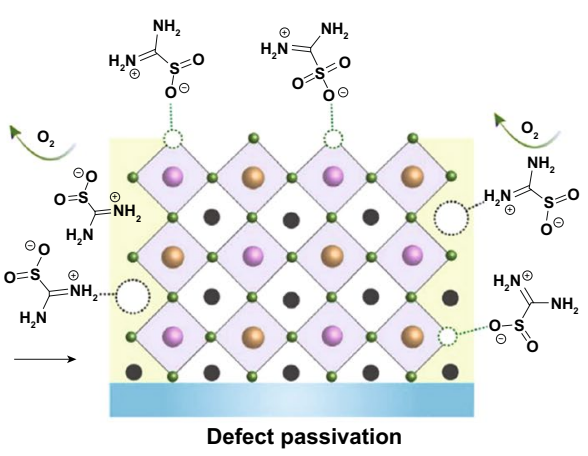

(f)

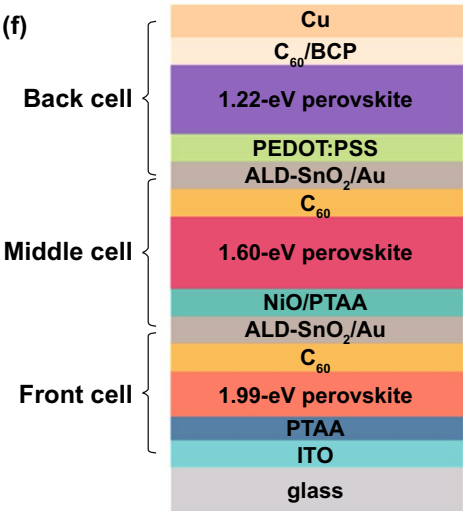

Fig. 6 a Schematic of the photoluminescence measurement and the chemical structure of carbazole-based HTL molecules, with R representing a methyl group for Me-4PACz. The number of 4 denotes the number of carbon atoms between the phosphonic anchoring group and the carbazole unit for Me-4PACz. b The FF loss mechanisms for PTAA and Me-4PACz PSCs based on the detailed balance analysis. Reproduced with permission from Ref. [10] Copyright 2021 AAAS. c Schematic illustration of suppressing $\mathrm{Sn}^{2+}$ oxidation and the passivation of halide and cation vacancy at the grain surface of mixed $\mathrm{Sn}-\mathrm{Pb}$ perovskite films enabled by FSA molecule. A-site represents the organic monovalent cations in the lattice. d Structure of all-perovskite tandem device with $1.22 \mathrm{eV}$ narrow-bandgap absorber and $1.77 \mathrm{eV}$ wide-bandgap absorber. e $J-V$ curves of the best control and FSA tandem devices with an aperture area of $1.05 \mathrm{~cm}^{2}$. Reproduced with permission from Ref. [9] Copyright 2020 Nature Publishing Group. f Device configuration of the all-perovskite triple-junction tandem device. Reproduced with permission from Ref. [65] Copyright 2020 American Chemical Society Publications

bottom cell and employing a self-assembled 1-butanethiol passivation layer on the perovskite surface. This strategy not only increased the carrier diffusion length but also stabilized the wide-bandgap perovskite phase, enabling a certified efficiency of $25.7 \%$. Similarly, Chen et al. used a nitrogen-assisted blading process to deposit hole transport layer and high-quality planarizing perovskite absorber that fully covered the rough silicon pyramids [61]. Moreover, a textured light-scattering layer was added to the perovskite top cell to reduce reflectance at the front surface, leading 
to an efficiency of $26.0 \%$ for textured Si-perovskite tandem devices.

\subsection{All-Perovskite Tandem Structure}

Compared to the Si-perovskite tandems, all-perovskite tandems exhibit additional advantages of low materials and fabrication costs because the bottom and top cells can be fabricated by the same preparation process without using specific equipment for other types of solar cells. The initial two-terminal all-perovskite tandems used $\mathrm{MAPbBr}_{3}$ and $\mathrm{MAPbI}_{3}$ as the wide-bandgap $(2.30 \mathrm{eV})$ and narrow-bandgap $(1.55 \mathrm{eV})$ absorbers, respectively, giving an efficiency of $10.8 \%[62]$.

After a few years of development, the efficiency of all-perovskite tandems has increased to over $24 \%$ via tailoring the bandgap alignment in devices. The mixed $\mathrm{Sn}-\mathrm{Pb}$ perovskites with a minimized bandgap of about $1.2 \mathrm{eV}$ are a promising candidate as the narrow-bandgap absorbers [63]. However, the spontaneous oxidation of $\mathrm{Sn}^{2+}$ to $\mathrm{Sn}^{4+}$ and $\mathrm{Sn}$ vacancy cause a high defect density in $\mathrm{Sn}-\mathrm{Pb}$ perovskites and thus a shorter carrier diffusion length compared to that of the full$\mathrm{Pb}$ counterpart, which lowers the charge transport efficiency in the thick narrow-bandgap absorber (over $1 \mu \mathrm{m}$ ). To overcome this challenge, Lin et al. used metallic $\mathrm{Sn}$ to reduce the $\mathrm{Sn}^{4+}$ impurities in perovskite precursors via a comproportionation reaction $\left(\mathrm{Sn}+\mathrm{Sn}^{4+} \rightarrow \mathrm{Sn}^{2+}\right)$ [64], reducing the defect density from $1.4 \times 10^{16}$ to $5.4 \times 10^{15} \mathrm{~cm}^{-3}$ and thereby prolonging the diffusion length from 0.75 to $2.99 \mu \mathrm{m}$ in $\mathrm{MA}_{0.3} \mathrm{FA}_{0.7} \mathrm{~Pb}_{0.5} \mathrm{Sn}_{0.5} \mathrm{I}_{3}$ perovskites. After integrating this narrow-bandgap $\mathrm{Sn}-\mathrm{Pb}$ PSCs $(1.22 \mathrm{eV})$ with the wide-bandgap PSCs $(1.77 \mathrm{eV})$, they obtained a high PCE of $24.8 \%$ for the tandem solar cells.

To further reduce the defect density in mixed $\mathrm{Sn}-\mathrm{Pb}$ perovskite, Lin et al. employed a strongly reductive surfaceanchoring molecules, formamidine sulfinic acid (FSA), to reduce the $\mathrm{Sn}^{4+}$ defects and passivate the surface ionic vacancy [9]. As shown in Fig. 6c, the sulfinic acid group could react with oxygen molecules and thus suppress the $\mathrm{Sn}^{2+}$ oxidation. Meanwhile, the $\mathrm{O}$ atoms of sulfinic group serving as an electron donor could form coordination bond with the under-coordinated $\mathrm{Sn}^{2+}$ or $\mathrm{Pb}^{2+}$ cations to passivate the surface halide vacancy. On the other hand, the formamidine group of FSA showing similar structure with FA cation could also passivate the surface A-site vacancy defects. Such synergistic effect of the surface-anchoring FSA improved the carrier recombination lifetime in $\mathrm{Sn}-\mathrm{Pb}$ perovskites by three times. Via employing this narrow-bandgap absorber into the monolithic tandem structure (Fig. 6d), they obtained a $V_{\mathrm{OC}}$ enhancement of about $60 \mathrm{mV}$ and a large PCE improvement from $22.7 \%$ to $24.7 \%$ for a $1.05-\mathrm{cm}^{2}$ all-perovskite tandem (Fig. 6e).

Furthermore, the solution-processed all-perovskite triplejunction tandem device with a PCE of over $20 \%$ was also reported [65]. This triple-junction solar cell contained a $1.99-\mathrm{eV}$ perovskite at the front cell, a $1.60-\mathrm{eV}$ perovskite at the middle cell, and a $1.22-\mathrm{eV}$ perovskite at the back cell (Fig. 6f). By developing compatible interconnecting layers with those solution-processed perovskite absorbers, a high $V_{\mathrm{OC}}$ of $2.8 \mathrm{~V}$ can be achieved, much higher than the value of all-perovskite double-junction solar cells.

\subsection{Lead-Free PSCs}

With the continuous movement of PSCs to commercialization, the toxicity of $\mathrm{Pb}$ element in perovskite absorber arouses the concern of environmental problems [66]. In recent years, a growing number of studies have aimed at developing the eco-friendly lead-free PSCs to directly avoid the use of lead in metal halide perovskite layers. So far, a number of lead-free perovskites based on tin (Sn), antimony ( $\mathrm{Sb}$ ), bismuth (Bi), titanium (Ti), germanium (Ge), and copper $(\mathrm{Cu})$ have been exploited for the application of solar cells [67-71].

Among all the lead-free perovskite materials, the most promising candidate is tin perovskite. Sn has a similar outer electronic structure $\left(\mathrm{ns}^{2} \mathrm{np}^{2}\right.$ ) and ionic radius to $\mathrm{Pb}$, enabling the complete replacement of lead in perovskite lattice without causing notable phase segregation. Furthermore, tin perovskites demonstrate some additional advantages: (1) ideal bandgap close to Shockley-Queisser limit $(1.3 \sim 1.4 \mathrm{eV})$, (2) low exciton binding energy (29 meV for $\mathrm{MASnI}_{3}$ and $62 \mathrm{meV}$ for $\mathrm{MAPbI}_{3}$ ), and (3) high charge carrier mobility $\left(\mu_{\mathrm{e}}\right.$ (electron mobility) $=2000 \mathrm{~cm}^{2} \mathrm{~V}^{-1} \mathrm{~s}^{-1}$ for $\mathrm{MASnI}_{3}$ and $60 \mathrm{~cm}^{2} \mathrm{~V}^{-1} \mathrm{~s}^{-1}$ for $\mathrm{MAPbI}_{3}$ ) [72, 73].

After a few years of development, the efficiency of tin PSCs underwent a rapid growth from 6 to 11\%-13\% owing to the careful design of antioxidant additives, mediating the crystallization rate, design of suitable A-site cations such as phenethylammonium (PEA) and 
pentafluorophen-oxyethylammonium (FOE), and constructing the oriented low-dimensional perovskite structure [22, $74,75]$. Currently, researchers mainly focus on the defect passivation and selection of suitable electron transport layers to minimize the $V_{\mathrm{OC}}$ loss in tin PSCs.

Nishimura et al. combined the bulk ethylammonium (EA) doping and surface edamine passivation to reduce the defect density of $\mathrm{FASnI}_{3}$ perovskite films by as much as 1 order of magnitude [21], which resulted in a high device $V_{\mathrm{OC}}$ of $0.84 \mathrm{~V}$. Furthermore, our group developed a template-growth technique assisted by an n-propylammonium iodide (PAI) salt to reduce the bulk defect density in the solution-processed $\mathrm{FASnI}_{3}$ films [24]. The PAI post-treatment increased the electron diffusion length from 70 to $180 \mathrm{~nm}$ and thereby reduced the recombination loss in $\mathrm{FASnI}_{3}$ absorbers due to the increased crystal orientation along (h00) directions (Fig. 7a), which enabled a significant $V_{\mathrm{OC}}$ enhancement of $200 \mathrm{mV}$ and a record certified PCE of $11.22 \%$ for tin PSCs (Fig. 7b). On the other side, Jiang et al. utilized indene- $\mathrm{C}_{60}$ bis-adduct (ICBA) with a high LUMO position as the electron transport layer to reduce the energy-level offset in tin PSCs [76]. They found that a shallower LUMO of $-3.74 \mathrm{eV}$ in ICBA than that $(-3.91 \mathrm{eV})$ of traditional [6, 6]-phenyl- $\mathrm{C}_{61}$-butyric acid methyl ester (PCBM) can upshift the quasi-Fermi level of electrons, approaching the $\mathrm{CBM}(-3.69 \mathrm{eV})$ of $\mathrm{FA}_{0.85} \mathrm{PEA}_{0.15} \mathrm{SnI}_{3}$ perovskite (PEA represents cation) and thus improving the quasi-Fermi level splitting and the maximum attainable $V_{\mathrm{OC}}$. Consequently, the ICBA-based tin PSCs obtained an ultra-high open-circuit voltage of $0.94 \mathrm{~V}$, about $300 \mathrm{mV}$ increasing compared to that of the PCBM-based cell, which enables a high PCE of $12.4 \%$. The detailed PV parameters of tin PSCs reported in 2020-2021 are summarized in Table 1.
Besides the tin PSCs, other types of lead-free PSCs based on wide-bandgap $\mathrm{Bi}$ and $\mathrm{Sb}$ perovskites also showed a large efficiency progress. Hu et al. fabricated the bulk-heterojunction perovskite active layers consisting of phase-separated $\mathrm{Cs}_{3} \mathrm{Bi}_{2} \mathrm{I}_{9}$ and $\mathrm{Ag}_{3} \mathrm{Bi}_{2} \mathrm{I}_{9}$ to improve the grain orientation and interface band alignment [77], achieving a record efficiency of about $3.6 \%$ with a high $V_{\mathrm{OC}}$ reaching $0.89 \mathrm{~V}$ for Bi-based PSCs. Singh et al. used the indacenodithiophenebased organic acceptor with Lewis base groups to improve the morphology of wide-bandgap $\mathrm{Cs}_{3} \mathrm{Sb}_{2} \mathrm{I}_{9}$ perovskites and enhance the electron-extraction ability in Sb-based PSCs [78], yielding a PCE of $3.25 \%$ for the inverted-structure device.

\section{Conclusions and Perspectives}

In 2020 and 2021, researchers from all over the word have reported exciting progress on the improvement of efficiency and stability of PSCs, and both the solar cells and modules have been demonstrated to pass the $85{ }^{\circ} \mathrm{C} / 85 \% \mathrm{RH}$ test. Furthermore, the entrepreneurs in China and other countries are pushing forward the large-scale production of PSCs. Microquanta Semiconductor has established a 5GW-capacity pilot manufacturing facility for perovskite solar products, which is the first production facility for perovskite PV in the world. At the same time, GCL New Energy is building its $100 \mathrm{MW}$-capacity production line in Kunshan for fabricating the $18 \%$-efficiency PSC modules with a decrease of $70 \%$ in production cost compared to that of the crystalline-Si. Oxford PV has recently increased the efficiency of perovskite-silicon tandem cells to $29.52 \%$ and announced that a $125 \mathrm{MW}$-capacity production line in Berlin is under (a)

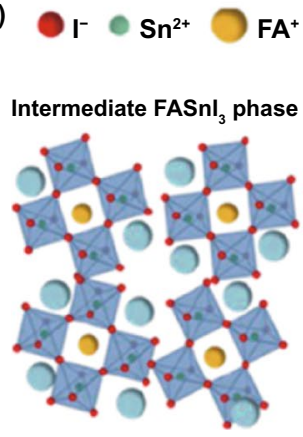

DMSO

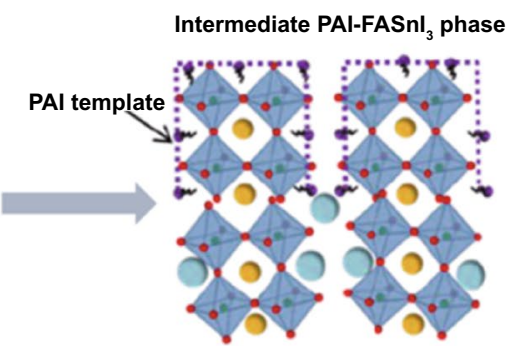

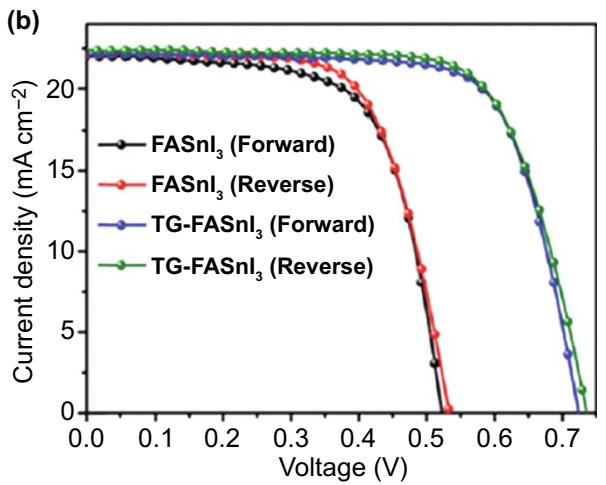

Fig. 7 a Possible mechanism of the template-growth process for FASnI ${ }_{3}$ perovskite film. $\mathbf{b} J$ - $V$ plots of the champion tin PSCs based on control and template-growth $\mathrm{FASnI}_{3}$ perovskite absorbers. Reproduced with permission from Ref. [24] Copyright 2020 The Royal Society of Chemistry 
Table 1 Photovoltaic parameters of the tin PSCs reported in 2020-2021, all the device efficiencies were measured in the third-party institutes or accredited test centers

\begin{tabular}{|c|c|c|c|c|c|c|}
\hline Perovskite & $J_{\mathrm{SC}}\left(\mathrm{mA} \mathrm{cm}^{-2}\right)$ & $V_{\mathrm{OC}}(\mathrm{V})$ & $\mathrm{FF}(\%)$ & PCE (\%) & Test Center & Refs \\
\hline $\mathrm{FASnI}_{3}$ & 21.14 & 0.61 & 73.4 & 9.39 & $\operatorname{AIST}^{a}$ (Japan) & {$[72]$} \\
\hline $\mathrm{Cs}_{0.1} \mathrm{FA}_{0.9} \mathrm{SnI}_{3}$ & 22.21 & 0.64 & 70.8 & 10.08 & Newport (USA) & {$[75]$} \\
\hline $\mathrm{PEA}_{0.15} \mathrm{FA}_{0.85} \mathrm{SnI}_{3}$ & 17.40 & 0.94 & 75.0 & 12.40 & $\operatorname{SIMIT}^{b}$ (China) & {$[76]$} \\
\hline $\mathrm{FOE}_{0.02} \mathrm{FA}_{0.98} \mathrm{SnI}_{3}$ & 21.95 & 0.64 & 72.5 & 10.16 & Newport (USA) & {$[74]$} \\
\hline $\mathrm{FA}_{0.75} \mathrm{MA}_{0.25} \mathrm{SnI}_{3}$ & 23.65 & 0.69 & 68.0 & 11.16 & KISTEC $^{c}$ (Japan) & {$[73]$} \\
\hline $\mathrm{PA}_{0.2} \mathrm{FA}_{0.8} \mathrm{SnI}_{3}$ & 22.01 & 0.70 & 73.3 & 11.22 & Newport (USA) & {$[24]$} \\
\hline
\end{tabular}

${ }^{a}$ AIST represents National Institute of Advanced Industrial Science and Technology

${ }^{b}$ SIMIT represents Shanghai Institute of Microsystem and Information Technology, Chinese Academy of Science

${ }^{c}$ KISTEC represents the Kanagawa Institute of Industrial Science and Technology

construction for producing the perovskite-based residential rooftop panels. Moreover, Wonder Solar Ltd. continues to develop the fully printable PSC modules and has demonstrated a $110 \mathrm{~m}^{2}$ power generation system outdoor. We hope that such collaboration between academia and PV industry can be further intensified to boost the commercialization of PSCs.

Although many efforts have been done, the significantly reduced efficiency upon solar module area scaling-up is still the main challenge to face for the commercialization of PSCs. As shown in Fig. 8, the PCE decreases to $19.6 \%$ when the aperture area increases from $0.1 \mathrm{~cm}^{2}$ to about $10 \mathrm{~cm}^{2}$, and further drops to $17.9 \%$ with the area approaching 1000

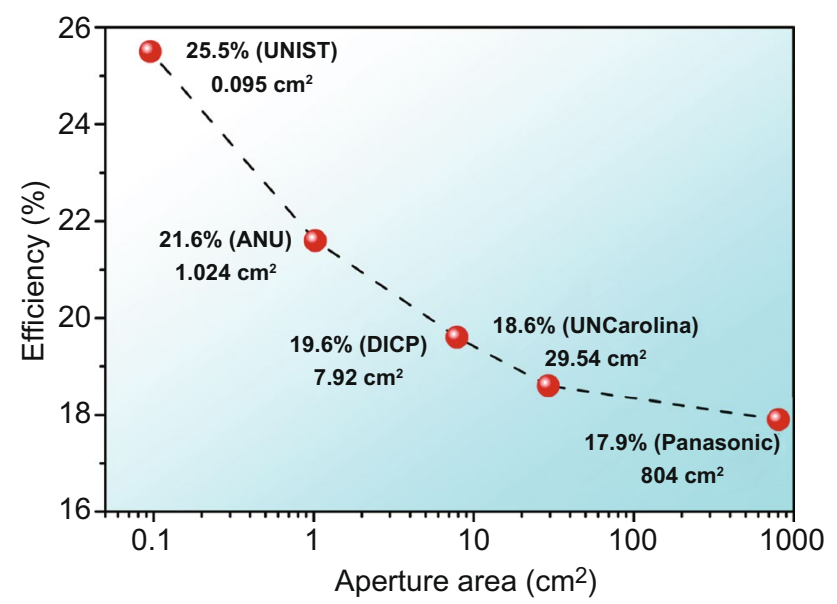

Fig. 8 The record efficiency of PSCs as a function of the aperture area in 2020. UNIST represents Ulsan National Institute of Science and Technology (Republic of Korea), ANU represents the Australian National University (Australia), DCIP represents Dalian Institute of Chemical Physics (China), UNCarolina represents the University of North Carolina at Chapel Hill (USA) $\mathrm{cm}^{2}$ for PSCs, which still lags far behind that of the crystalline silicon cells $\left(26.7 \%\right.$ at $79.0 \mathrm{~cm}^{2}$ and $24.4 \%$ at 13,177 $\mathrm{cm}^{2}$ ) [6]. Therefore, intensive works should be conducted to precisely control the uniformity of the crystallization process in large-area perovskite films. Also, the fundamental mechanisms relative to the PCE loss in PSC modules should be further studied. On the other side, realizing open-air and high-speed coating of perovskite films is also important for reducing the module manufacturing cost in production line, and some related studies have been done. Deng et al. first demonstrated a tailoring solvent coordination method to blade large-area perovskite films with a high speed of $5.9 \mathrm{~m} \mathrm{~min}^{-1}$ under ambient condition. They used the lowvolatile, coordinating solvents for $\mathrm{Pb}^{2+}$ components to obtain high-quality perovskite films, yielding a module efficiency of $16.4 \%$ [5]. Recently, Rolston et al. reported an open-air rapid spray plasma processing method that enabled a record coating speed of $12 \mathrm{~m} \mathrm{~min}^{-1}$ without any post-annealing for CsFA-based perovskite films, which resulted in a module efficiency of $15.5 \%$ [79].

Furthermore, recent study showed that organic solvents used in the mass production of PSC modules such as $\mathrm{N}, \mathrm{N}$-dimethylformamide (DMF), N,N-dimethylacetamide (DMAC), N-methyl-2-pyrrolidone (NMP), and gammabutyrolactone (GBL) are toxic to the human reproductive systems [80]. Therefore, development of green solvent systems or the solvent-free deposition technology for fabricating large-area perovskite film will be an important research topic in the future $[81,82]$.

Besides the efficiency, more and more attention has been paid to the long-term stability of PSC modules. In 2020, Microquanta company announced that its mass-produced 
PSC module has passed the strict stability test according to the International Electro technical Commission (IEC) standards, the $20 \mathrm{~cm}^{2}$ perovskite module underwent $3000 \mathrm{~h}$ damp heat test without degradation, and showed an efficiency loss less than $2 \%$ after UV preconditioning test, associated with a product lifetime of over 25 years. Also, Utmo Light Ltd. reported that their PSC mini-module passed the IEC test with a stabilized efficiency of over $20 \%$, which is a milestone for PSCs toward the practical use. Recently, Okinawa Institute of Science and Technology Graduate University (OIST, Japan) reported over 1100-h operational lifetime for a $10 \times 10 \mathrm{~cm}^{2}$ solar module [83]. Although many research groups and companies claimed that their devices have passed IEC standard test, there are still some stability issues needed to be addressed at the next stage, one important thing is the cell encapsulation technology. A well-designed encapsulation of PSCs should not only block the outside moisture and oxygen effectively, but also suppress the escape of volatile products and lead from the decomposed organic hybrid perovskite layer [84]. In this regard, we believe that a growing number of studies will move to exploit such multifunctional encapsulation materials in the future.

So far, a series of stability tests custom-made for PSCs have been proposed [85], and we therefore suggest a standardized stability test for PSCs and encourage the researchers to measure the module stability at an authorized third-party test center to increase the credibility of their results.

Development of highly efficient lead-free PSCs is also an alternative choice to extend their application range in the PV markets, especially for the indoor power generation like wearable power sources that have a strict limit on lead content [86-88]. It was demonstrated that tin PSCs could be the next generation of PSCs for realizing over $20 \%$ efficiency and the strategies for their mass production have been investigated [20]. Moreover, developing reducing solvent system for $\mathrm{Sn}^{2+}$ precursor, constructing antioxidant capping layer during the crystallization of tin perovskite film, and the investigation of compatible device encapsulation approaches also led to a large stability improvement of tin PSCs $[89,90]$. In our opinion, the application of tin PSCs for indoor PV products could be prior to lead PSCs when their efficiencies reach over $15 \%$.

Acknowledgements This work was supported by the National Natural Science Foundation of China (Grant Nos. 11834011 and 12074245). Y. B. Q. acknowledges the support from the Energy
Materials and Surface Sciences Unit of the Okinawa Institute of Science and Technology Graduate University.

Open Access This article is licensed under a Creative Commons Attribution 4.0 International License, which permits use, sharing, adaptation, distribution and reproduction in any medium or format, as long as you give appropriate credit to the original author(s) and the source, provide a link to the Creative Commons licence, and indicate if changes were made. The images or other third party material in this article are included in the article's Creative Commons licence, unless indicated otherwise in a credit line to the material. If material is not included in the article's Creative Commons licence and your intended use is not permitted by statutory regulation or exceeds the permitted use, you will need to obtain permission directly from the copyright holder. To view a copy of this licence, visit http://creativecommons.org/ licenses/by/4.0/.

\section{References}

1. Y. Wang, T. Wu, J. Barbaud, W. Kong, D. Cui et al., Stabilizing heterostructures of soft perovskite semiconductors. Science 365(6454), 687-691 (2019). https://doi.org/10.1126/ science.aax 8018

2. Q. Jiang, Y. Zhao, X. Zhang, X. Yang, Y. Chen et al., Surface passivation of perovskite film for efficient solar cells. Nat. Photonics 13(7), 460-466 (2019). https://doi.org/10.1038/ s41566-019-0398-2

3. E.H. Jung, N.J. Jeon, E.Y. Park, C.S. Moon, T.J. Shin et al., Efficient, stable and scalable perovskite solar cells using poly(3-hexylthiophene). Nature 567(7749), 511-515 (2019). https://doi.org/10.1038/s41586-019-1036-3

4. T. Wu, Y. Wang, Z. Dai, D. Cui, T. Wang et al., Efficient and stable $\mathrm{CsPbI}_{3}$ solar cells via regulating lattice distortion with surface organic terminal groups. Adv. Mater. 31(24), 1900605 (2019). https://doi.org/10.1002/adma.201900605

5. Y. Deng, C.H. Van Brackle, X. Dai, J. Zhao, B. Chen et al., Tailoring solvent coordination for high-speed, room-temperature blading of perovskite photovoltaic films. Sci. Adv. 5(12), eaax7537 (2019). https://doi.org/10.1126/sciadv.aax7537

6. M. Green, E. Dunlop, J. Hohl Ebinger, M. Yoshita, N. Kopidakis et al., Solar cell efficiency tables (version 57). Prog. Photovoltaics 29(1), 3-15 (2021). https://doi.org/10.1002/pip. 3371

7. X. Luo, T. Wu, Y. Wang, X. Lin, H. Su et al., Progress of all-perovskite tandem solar cells: the role of narrow-bandgap absorbers. Sci. China Chem. 64(2), 218-227 (2021). https:// doi.org/10.1007/s11426-020-9870-4

8. S. Gu, R. Lin, Q. Han, Y. Gao, H. Tan et al., Tin and mixed lead-tin halide perovskite solar cells: progress and their application in tandem solar cells. Adv. Mater. 32(27), 1907392 (2020). https://doi.org/10.1002/adma.201907392

9. K. Xiao, R. Lin, Q. Han, Y. Hou, Z. Qin et al., All-perovskite tandem solar cells with $24.2 \%$ certified efficiency and area over $1 \mathrm{~cm}^{2}$ using surface-anchoring zwitterionic antioxidant. 
Nat. Energy 5(11), 870-880 (2020). https://doi.org/10.1038/ s41560-020-00705-5

10. A. Al Ashouri, E. Köhnen, B. Li, A. Magomedov, H. Hempel et al., Monolithic perovskite/silicon tandem solar cell with $>29 \%$ efficiency by enhanced hole extraction. Science 370(6522), 1300-1309 (2020). https://doi.org/10.1126/scien ce.abd4016

11. H. Su, T. Wu, D. Cui, X. Lin, X. Luo et al., The application of graphene derivatives in perovskite solar cells. Small Methods 4(10), 2000507 (2020). https://doi.org/10.1002/smtd.20200 0507

12. S. Wu, R. Chen, S. Zhang, B.H. Babu, Y. Yue et al., A chemically inert bismuth interlayer enhances long-term stability of inverted perovskite solar cells. Nat. Commun. 10(1), 1161 (2019). https://doi.org/10.1038/s41467-019-09167-0

13. N. Arora, M.I. Dar, A. Hinderhofer, N. Pellet, F. Schreiber et al., Perovskite solar cells with $\mathrm{CuSCN}$ hole extraction layers yield stabilized efficiencies greater than $20 \%$. Science 358(6364), 768-771 (2017). https://doi.org/10.1126/science. aam5655

14. H. Chen, Q. Wei, M.I. Saidaminov, F. Wang, A. Johnston et al., Efficient and stable inverted perovskite solar cells incorporating secondary amines. Adv. Mater. 31(46), 1903559 (2019). https://doi.org/10.1002/adma.201903559

15. S. Bai, P. Da, C. Li, Z. Wang, Z. Yuan et al., Planar perovskite solar cells with long-term stability using ionic liquid additives. Nature 571(7764), 245-250 (2019). https://doi.org/10.1038/ s41586-019-1357-2

16. D. Wei, F. Ma, R. Wang, S. Dou, P. Cui et al., Ion-migration inhibition by the cation-pi interaction in perovskite materials for efficient and stable perovskite solar cells. Adv. Mater. 30(31), 1707583 (2018). https://doi.org/10.1002/adma.20170 7583

17. L. Liu, S. Huang, Y. Lu, P. Liu, Y. Zhao et al., Grain-boundary "patches" by in situ conversion to enhance perovskite solar cells stability. Adv. Mater. 30(29), 1800544 (2018). https:// doi.org/10.1002/adma.201800544

18. N. Li, S. Tao, Y. Chen, X. Niu, C.K. Onwudinanti et al., Cation and anion immobilization through chemical bonding enhancement with fluorides for stable halide perovskite solar cells. Nat. Energy 4(5), 408-415 (2019). https://doi.org/10.1038/ s41560-019-0382-6

19. A. Mei, Y. Sheng, Y. Ming, Y. Hu, Y. Rong et al., Stabilizing perovskite solar cells to IEC61215:2016 standards with over 9,000-h operational tracking. Joule 4(12), 2646-2660 (2020). https://doi.org/10.1016/j.joule.2020.09.010

20. T. Wu, X. Liu, X. Luo, X. Lin, D. Cui et al., Lead-free tin perovskite solar cells. Joule 5(4), 863-886 (2021). https://doi. org/10.1016/j.joule.2021.03.001

21. K. Nishimura, M.A. Kamarudin, D. Hirotani, K. Hamada, Q. Shen et al., Lead-free tin-halide perovskite solar cells with 13\% efficiency. Nano Energy 74, 104858 (2020). https://doi. org/10.1016/j.nanoen.2020.104858

22. C. Wang, Y. Zhang, F. Gu, Z. Zhao, H. Li et al., Illumination durability and high-efficiency Sn-based perovskite solar cell under coordinated control of phenylhydrazine and halogen ions. Matter 4(2), 709-721 (2021). https://doi.org/10.1016/j. matt.2020.11.012

23. X. Li, F. Zhang, H. He, J.J. Berry, K. Zhu et al., On-device lead sequestration for perovskite solar cells. Nature 578(7796), 555-558 (2020). https://doi.org/10.1038/s41586-020-2001-X

24. X. Liu, T. Wu, J.Y. Chen, X. Meng, X. He et al., Templated growth of FASnI(3) crystals for efficient tin perovskite solar cells. Energy Environ. Sci. 13(9), 2896-2902 (2020). https:// doi.org/10.1039/d0ee01845g

25. C. Zhang, Y. Wang, X. Lin, T. Wu, Q. Han et al., Effects of A site doping on the crystallization of perovskite films. J. Mater. Chem. A 9(3), 1372-1394 (2021). https://doi.org/10. 1039/D0TA08656H

26. C. Shen, Y. Wu, S. Zhang, T. Wu, H. Tian et al., Stabilizing formamidinium lead iodide perovskite by sulfonyl-functionalized phenethylammonium salt via crystallization control and surface passivation. Sol. RRL 4(5), 2000069 (2020). https:// doi.org/10.1002/solr.202000069

27. G. Kim, H. Min, K.S. Lee, D.Y. Lee, S.M. Yoon et al., Impact of strain relaxation on performance of $\alpha$-formamidinium lead iodide perovskite solar cells. Science 370(6512), 108-112 (2020). https://doi.org/10.1126/science.abc4417

28. H. Min, M. Kim, S.U. Lee, H. Kim, G. Kim et al., Efficient, stable solar cells by using inherent bandgap of $\alpha$-phase formamidinium lead iodide. Science 366(6466), 749-753 (2019). https://doi.org/10.1126/science.aay7044

29. J. Jeong, M. Kim, J. Seo, H. Lu, P. Ahlawat et al., Pseudohalide anion engineering for $\alpha-\mathrm{FAPbI}_{3}$ perovskite solar cells. Nature 592(7854), 381-385 (2021). https://doi.org/10.1038/ s41586-021-03406-5

30. H. Fan, F. Li, P. Wang, Z. Gu, J.H. Huang et al., Methylamineassisted growth of uniaxial-oriented perovskite thin films with millimeter-sized grains. Nat. Commun. 11(1), 5402 (2020). https://doi.org/10.1038/s41467-020-19199-6

31. Q. Dong, Y. Fang, Y. Shao, P. Mulligan, J. Qiu et al., Electron-hole diffusion lengths $>175 \mu \mathrm{m}$ in solution-grown $\mathrm{CH}_{3} \mathrm{NH}_{3} \mathrm{PbI}_{3}$ single crystals. Science 347(6225), 967-970 (2015). https://doi.org/10.1126/science.aaa5760

32. T. Wu, Y. Wang, X. Li, Y. Wu, X. Meng et al., Efficient defect passivation for perovskite solar cells by controlling the electron density distribution of donor- $\pi$-acceptor molecules. Adv. Energy Mater. 9(17), 1803766 (2019). https://doi.org/10.1002/ aenm.201803766

33. L.K. Ono, S. Liu, Y.B. Qi, Reducing detrimental defects for high-performance metal halide perovskite solar cells. Angew. Chem. Int. Ed. 59(17), 6676-6698 (2020). https://doi.org/10. 1002/anie.201905521

34. B. Chen, P.N. Rudd, S. Yang, Y. Yuan, J. Huang, Imperfections and their passivation in halide perovskite solar cells. Chem. Soc. Rev. 48(14), 3842-3867 (2019). https://doi.org/10.1039/ C8CS00853A

35. X. Zheng, Y. Hou, C. Bao, J. Yin, F. Yuan et al., Managing grains and interfaces via ligand anchoring enables $22.3 \%$-efficiency inverted perovskite solar cells. Nat. Energy 5(2), 131140 (2020). https://doi.org/10.1038/s41560-019-0538-4 
36. Q. Wang, Q. Dong, T. Li, A. Gruverman, J. Huang, Thin insulating tunneling contacts for efficient and water-resistant perovskite solar cells. Adv. Mater. 28(31), 6734-6739 (2016). https://doi.org/10.1002/adma.201600969

37. J. Peng, D. Walter, Y. Ren, M. Tebyetekerwa, Y. Wu et al., Nanoscale localized contacts for high fill factors in polymerpassivated perovskite solar cells. Science 371(6527), 390-395 (2021). https://doi.org/10.1126/science.abb8687

38. M. Jeong, I.W. Choi, E.M. Go, Y. Cho, M. Kim et al., Stable perovskite solar cells with efficiency exceeding $24.8 \%$ and 0.3-V voltage loss. Science 369(6511), 1615-1620 (2020). https://doi.org/10.1126/science.abb7167

39. J.J. Yoo, G. Seo, M.R. Chua, T.G. Park, Y. Lu et al., Efficient perovskite solar cells via improved carrier management. Nature 590(7847), 587-593 (2021). https://doi.org/10.1038/ s41586-021-03285-w

40. Y. Yuan, J. Huang, Ion migration in organometal trihalide perovskite and its impact on photovoltaic efficiency and stability. Acc. Chem. Res. 49(2), 286-293 (2016). https://doi.org/10. 1021/acs.accounts.5b00420

41. P. Delugas, C. Caddeo, A. Filippetti, A. Mattoni, Thermally activated point defect diffusion in methylammonium lead trihalide: anisotropic and ultrahigh mobility of iodine. J. Phys. Chem. Lett. 7(13), 2356-2361 (2016). https://doi.org/10.1021/ acs.jpclett.6b00963

42. M.I. Saidaminov, J. Kim, A. Jain, R. Quintero-Bermudez, H. Tan et al., Suppression of atomic vacancies via incorporation of isovalent small ions to increase the stability of halide perovskite solar cells in ambient air. Nat. Energy 3(8), 648-654 (2018). https://doi.org/10.1038/s41560-018-0192-2

43. Y.H. Lin, N. Sakai, P. Da, J. Wu, H.C. Sansom et al., A piperidinium salt stabilizes efficient metal-halide perovskite solar cells. Science 369(6499), 96-102 (2020). https://doi.org/10. 1126/science.aba1628

44. S.G. Motti, D. Meggiolaro, A.J. Barker, E. Mosconi, C.A.R. Perini et al., Controlling competing photochemical reactions stabilizes perovskite solar cells. Nat. Photonics 13(8), 532-539 (2019). https://doi.org/10.1038/s41566-019-0435-1

45. K. Wang, J. Liu, J. Yin, E. Aydin, G.T. Harrison et al., Defect passivation in perovskite solar cells by cyano-based $\pi$-conjugated molecules for improved performance and stability. Adv. Funct. Mater. 30(35), 2002861 (2020). https://doi. org/10.1002/adfm.202002861

46. S. Xiong, J. Song, J. Yang, J. Xu, M. Zhang et al., Defectpassivation using organic dyes for enhanced efficiency and stability of perovskite solar cells. Sol. RRL 4(5), 1900529 (2020). https://doi.org/10.1002/solr.201900529

47. Z. Yang, J.J. Dou, S. Kou, J.L. Dang, Y.Q. Ji et al., Multifunctional phosphorus-containing lewis acid and base passivation enabling efficient and moisture-stable perovskite solar cells. Adv. Funct. Mater. 30(15), 1910710 (2020). https://doi.org/ 10.1002/adfm.201910710

48. Z. Liu, L. Qiu, L.K. Ono, S. He, Z. Hu et al., A holistic approach to interface stabilization for efficient perovskite solar modules with over 2,000-hour operational stability.
Nat. Energy 5(8), 596-604 (2020). https://doi.org/10.1038/ s41560-020-0653-2

49. Y. Sha, E. Bi, Y. Zhang, P. Ru, W. Kong et al., A scalable integrated dopant-free heterostructure to stabilize perovskite solar cell modules. Adv. Energy Mater. 11(5), 2003301 (2021). https://doi.org/10.1002/aenm.202003301

50. B. Hailegnaw, S. Kirmayer, E. Edri, G. Hodes, D. Cahen, Rain on methylammonium lead iodide based perovskites: possible environmental effects of perovskite solar cells. J. Phys. Chem. Lett. 6(9), 1543-1547 (2015). https://doi.org/10.1021/acs.jpcle tt.5b00504

51. Y. Jiang, L. Qiu, E.J. Juarez-Perez, L.K. Ono, Z. Hu et al., Reduction of lead leakage from damaged lead halide perovskite solar modules using self-healing polymer-based encapsulation. Nat. Energy 4(7), 585-593 (2019). https://doi.org/10. 1038/s41560-019-0406-2

52. S. Chen, Y. Deng, H. Gu, S. Xu, S. Wang et al., Trapping lead in perovskite solar modules with abundant and low-cost cation-exchange resins. Nat. Energy 5(12), 1003-1011 (2020). https://doi.org/10.1038/s41560-020-00716-2

53. D. Kim, H.J. Jung, I.J. Park, B.W. Larson, S.P. Dunfield et al., Efficient, stable silicon tandem cells enabled by anionengineered wide-bandgap perovskites. Science 368(6487), 155-160 (2020). https://doi.org/10.1126/science.aba3433

54. B. Chen, Z. Yu, K. Liu, X. Zheng, Y. Liu et al., Grain engineering for perovskite/silicon monolithic tandem solar cells with efficiency of $25.4 \%$. Joule 3(1), 177-190 (2019). https:// doi.org/10.1016/j.joule.2018.10.003

55. Y. Wang, X. Liu, T. Zhang, X. Wang, M. Kan et al., The role of dimethylammonium iodide in $\mathrm{CsPb}_{3}$ perovskite fabrication: additive or dopant? Angew. Chem. Int. Ed. 58(46), 1669116696 (2019). https://doi.org/10.1002/anie.201910800

56. S. Gharibzadeh, B. Abdollahi Nejand, M. Jakoby, T. Abzieher, D. Hauschild et al., Record open-circuit voltage wide-bandgap perovskite solar cells utilizing 2D/3D perovskite heterostructure. Adv. Energy Mater. 9(21), 1803699 (2019). https://doi. org/10.1002/aenm.201803699

57. J. Xu, C.C. Boyd, Z.J. Yu, A.F. Palmstrom, D.J. Witter et al., Triple-halide wide-band gap perovskites with suppressed phase segregation for efficient tandems. Science 367(6482), 1097-1104 (2020). https://doi.org/10.1126/science.aaz5074

58. I.L. Braly, R.J. Stoddard, A. Rajagopal, A.R. Uhl, J.K. Katahara et al., Current-induced phase segregation in mixed halide hybrid perovskites and its impact on two-terminal tandem solar cell design. ACS Energy Lett. 2(8), 1841-1847 (2017). https://doi.org/10.1021/acsenergylett.7b00525

59. M.C. Brennan, S. Draguta, P.V. Kamat, M. Kuno, Lightinduced anion phase segregation in mixed halide perovskites. ACS Energy Lett. 3(1), 204-213 (2018). https://doi.org/10. 1021/acsenergylett.7b01151

60. Y. Hou, E. Aydin, M. De. Bastiani, C. Xiao, F.H. Isikgor et al., Efficient tandem solar cells with solution-processed perovskite on textured crystalline silicon. Science 367(6482), 1135-1140 (2020). https://doi.org/10.1126/science.aaz3691

61. B. Chen, Z.J. Yu, S. Manzoor, S. Wang, W. Weigand et al., Blade-coated perovskites on textured silicon for $26 \%$-efficient 
monolithic perovskite/silicon tandem solar cells. Joule 4(4), 850-864 (2020). https://doi.org/10.1016/j.joule.2020.01.008

62. J.H. Heo, S.H. Im, $\mathrm{CH}_{3} \mathrm{NH}_{3} \mathrm{PbBr}_{3}-\mathrm{CH}_{3} \mathrm{NH}_{3} \mathrm{PbI}_{3}$ perovskiteperovskite tandem solar cells with exceeding $2.2 \mathrm{~V}$ open circuit voltage. Adv. Mater. 28(25), 5121-5125 (2016). https:// doi.org/10.1002/adma.201501629

63. J. Im, C.C. Stoumpos, H. Jin, A.J. Freeman, M.G. Kanatzidis, Antagonism between spin-orbit coupling and steric effects causes anomalous band gap evolution in the perovskite photovoltaic materials $\mathrm{CH}_{3} \mathrm{NH}_{3} \mathrm{Sn}_{1-\mathrm{x}} \mathrm{Pb}_{\mathrm{x}} \mathrm{I}_{3}$. J. Phys. Chem. Lett. 6(17), 3503-3509 (2015). https://doi.org/10.1021/acs.jpclett. $5 b 01738$

64. R. Lin, K. Xiao, Z. Qin, Q. Han, C. Zhang et al., Monolithic all-perovskite tandem solar cells with $24.8 \%$ efficiency exploiting comproportionation to suppress $\mathrm{Sn}(\mathrm{II})$ oxidation in precursor ink. Nat. Energy 4(10), 864-873 (2019). https://doi. org/10.1038/s41560-019-0466-3

65. K. Xiao, J. Wen, Q. Han, R. Lin, Y. Gao et al., Solution-processed monolithic all-perovskite triple-junction solar cells with efficiency exceeding 20\%. ACS Energy Lett. 5(9), 28192826 (2020). https://doi.org/10.1021/acsenergylett.0c01184

66. M.G. Ju, M. Chen, Y. Zhou, J. Dai, L. Ma et al., Toward ecofriendly and stable perovskite materials for photovoltaics. Joule 2(7), 1231-1241 (2018). https://doi.org/10.1016/j.joule. 2018.04.026

67. B. Saparov, F. Hong, J.P. Sun, H.S. Duan, W. Meng et al., Thin-film preparation and characterization of $\mathrm{Cs}_{3} \mathrm{Sb}_{2} \mathrm{I}_{9}$ : a leadfree layered perovskite semiconductor. Chem. Mater. 27(16), 5622-5632 (2015). https://doi.org/10.1021/acs.chemmater. $5 \mathrm{~b} 01989$

68. D. Cortecchia, H.A. Dewi, J. Yin, A. Bruno, S. Chen et al., Lead-free $\mathrm{MA}_{2} \mathrm{CuCl}_{\mathrm{x}} \mathrm{Br}_{4-} \mathrm{x}$ hybrid perovskites. Inorg. Chem. 55(3), 1044-1052 (2016). https://doi.org/10.1021/acs.inorg chem.5b01896

69. T. Krishnamoorthy, H. Ding, C. Yan, W.L. Leong, T. Baikie et al., Lead-free germanium iodide perovskite materials for photovoltaic applications. J. Mater. Chem. A 3(47), 2382923832 (2015). https://doi.org/10.1039/C5TA05741H

70. Z. Shao, T. Le. Mercier, M.B. Madec, T. Pauporté, Exploring $\mathrm{AgBi}_{\mathrm{x}} \mathrm{I}_{3 \mathrm{x}+1}$ semiconductor thin films for lead-free perovskite solar cells. Mater. Design 141, 81-87 (2018). https://doi.org/ 10.1016/j.matdes.2017.12.036

71. S. Öz, J.C. Hebig, E. Jung, T. Singh, A. Lepcha et al., Zerodimensional $\left(\mathrm{CH}_{3} \mathrm{NH}_{3}\right)_{3} \mathrm{Bi}_{2} \mathrm{I}_{9}$ perovskite for optoelectronic applications. Sol. Energy Mater. Sol. C 158, 195-201 (2016). https://doi.org/10.1016/j.solmat.2016.01.035

72. T. Wu, X. Liu, X. He, Y. Wang, X. Meng et al., Efficient and stable tin-based perovskite solar cells by introducing $\pi$-conjugated Lewis base. Sci. China Chem. 63(1), 107-115 (2020). https://doi.org/10.1007/s11426-019-9653-8

73. T. Nakamura, S. Yakumaru, M.A. Truong, K. Kim, J. Liu et al., $\mathrm{Sn}(\mathrm{iv})$-free tin perovskite films realized by in situ $\mathrm{Sn}(0)$ nanoparticle treatment of the precursor solution. Nat. Commun. 11(1), 3008 (2020). https://doi.org/10.1038/ s41467-020-16726-3
74. X. Meng, Y. Wang, J. Lin, X. Liu, X. He et al., Surface-controlled oriented growth of $\mathrm{FASnI}_{3}$ crystals for efficient leadfree perovskite solar cells. Joule 4(4), 902-912 (2020). https:// doi.org/10.1016/j.joule.2020.03.007

75. X. Liu, Y. Wang, T. Wu, X. He, X. Meng et al., Efficient and stable tin perovskite solar cells enabled by amorphouspolycrystalline structure. Nat. Commun. 11(1), 2678 (2020). https://doi.org/10.1038/s41467-020-16561-6

76. X. Jiang, F. Wang, Q. Wei, H. Li, Y. Shang et al., Ultra-high open-circuit voltage of tin perovskite solar cells via an electron transporting layer design. Nat. Commun. 11(1), 1245 (2020). https://doi.org/10.1038/s41467-020-15078-2

77. W. Hu, X. He, Z. Fang, W. Lian, Y. Shang et al., Bulk heterojunction gifts bismuth-based lead-free perovskite solar cells with record efficiency. Nano Energy 68, 104362 (2020). https://doi.org/10.1016/j.nanoen.2019.104362

78. A. Singh, P.T. Lai, A. Mohapatra, C.Y. Chen, H.W. Lin et al., Panchromatic heterojunction solar cells for $\mathrm{Pb}$-free all-inorganic antimony based perovskite. Chem. Eng. J. 419, 129424 (2021). https://doi.org/10.1016/j.cej.2021.129424

79. N. Rolston, W.J. Scheideler, A.C. Flick, J.P. Chen, H. Elmaraghi et al., Rapid open-air fabrication of perovskite solar modules. Joule 4(12), 2675-2692 (2020). https://doi.org/10. 1016/j.joule.2020.11.001

80. R. Vidal, J.A. Alberola-Borràs, S.N. Habisreutinger, J.L. Gimeno-Molina, D.T. Moore et al., Assessing health and environmental impacts of solvents for producing perovskite solar cells. Nat. Sustain. 4(3), 277-285 (2021). https://doi.org/10. 1038/s41893-020-00645-8

81. G. Tong, D.Y. Son, L.K. Ono, Y. Liu, Y. Hu et al., Scalable fabrication of $>90 \mathrm{~cm}^{2}$ perovskite solar modules with $>1000 \mathrm{~h}$ operational stability based on the intermediate phase strategy. Adv. Energy Mater. 11(10), 2003712 (2021). https://doi.org/ 10.1002/aenm.202003712

82. H. Chen, F. Ye, W. Tang, J. He, M. Yin et al., A solvent- and vacuum-free route to large-area perovskite films for efficient solar modules. Nature 550(7674), 92-95 (2017). https://doi. org/10.1038/nature23877

83. X. Cao, G. Zhang, Y. Cai, L. Jiang, X. He et al., All green solvents for fabrication of $\mathrm{CsPbBr}_{3}$ films for efficient solar cells guided by the hansen solubility theory. Sol. RRL 4(4), 2000008 (2020). https://doi.org/10.1002/solr.202000008

84. L. Shi, M.P. Bucknall, T.L. Young, M. Zhang, L. Hu et al., Gas chromatography-mass spectrometry analyses of encapsulated stable perovskite solar cells. Science 368(6497), eaba2412 (2020). https://doi.org/10.1126/science.aba2412

85. M.V. Khenkin, E.A. Katz, A. Abate, G. Bardizza, J.J. Berry et al., Consensus statement for stability assessment and reporting for perovskite photovoltaics based on isos procedures. Nat. Energy 5(1), 35-49 (2020). https://doi.org/10.1038/ s41560-019-0529-5

86. M. Konstantakou, T. Stergiopoulos, A critical review on tin halide perovskite solar cells. J. Mater. Chem. A 5(23), 11518 11549 (2017). https://doi.org/10.1039/C7TA00929A

87. E.W.G. Diau, E. Jokar, M. Rameez, Strategies to improve performance and stability for tin-based perovskite solar cells. 
ACS Energy Lett. 4(8), 1930-1937 (2019). https://doi.org/10. 1021/acsenergylett.9b01179

88. J. Cao, F. Yan, Recent progress in tin-based perovskite solar cells. Energy Environ. Sci. 14(3), 1286-1325 (2021). https:// doi.org/10.1039/D0EE04007J

89. X. Meng, T. Wu, X. Liu, X. He, T. Noda et al., Highly reproducible and efficient fasni3 perovskite solar cells fabricated with volatilizable reducing solvent. J. Phys. Chem. Lett. 11(8), 2965-2971 (2020). https://doi.org/10.1021/acs.jpclett.0c00923

90. X. He, T. Wu, X. Liu, Y. Wang, X. Meng et al., Highly efficient tin perovskite solar cells achieved in a wide oxygen concentration range. J. Mater. Chem. A 8(5), 2760-2768 (2020). https://doi.org/10.1039/C9TA13159K 